Supporting Information

\title{
Azo(xy) vs. Aniline Selectivity in Catalytic \\ Nitroarene Reduction by Intermetallics: Experiments \\ and Simulations
}

Carena L. Daniels,' Da-Jiang Liu,*2 Marquix A. S. Adamson,' Megan Knobeloch,' and Javier Vela $^{*, 2}$

'Department of Chemistry, Iowa State University, Ames, Iowa 50011, 'US DOE Ames

Laboratory, Iowa State University, Ames, Iowa 50011, United States

*e-mail: vela@iastate.edu, phone: 515-294-5536

*e-mail: dajiang@ameslab.gov, phone: 515-294-9927 
Scheme S1. Synthesis of binary intermetallic $\mathrm{Pd}_{2} \mathrm{Ge}, \mathrm{Pd}_{2} \mathrm{Sn}, \mathrm{Pd}_{3} \mathrm{Sn}_{2}, \mathrm{PtSn}(\mathrm{a}), \mathrm{Pd}_{3} \mathrm{~Pb}$ (b), and $\mathrm{Pd}_{x} \mathrm{Sn}_{1: x} / \mathrm{C}$ alloy (c) nanoparticles.

(a)

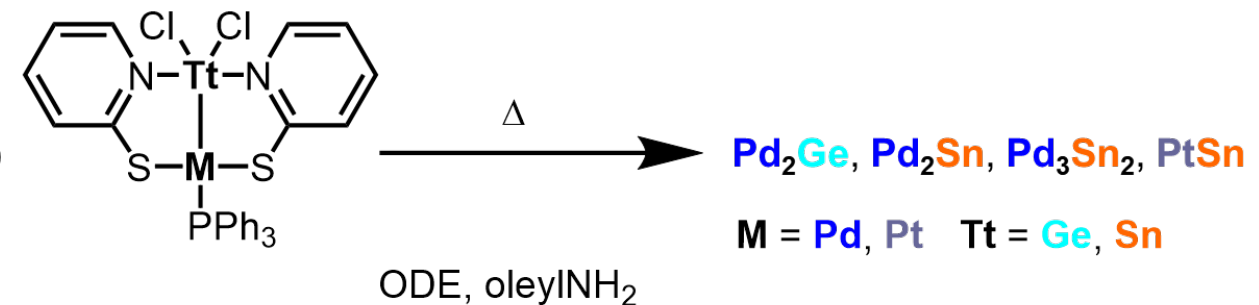

(b) $3 \mathrm{PdBr}_{2}+\mathrm{PbBr}_{2} \frac{\text { oleic acid }}{170{ }^{\circ} \mathrm{C}, 0.5 \mathrm{~h}} \mathrm{Pd}_{3} \mathrm{~Pb}$

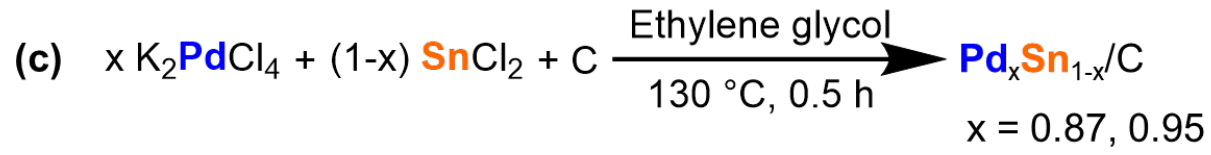




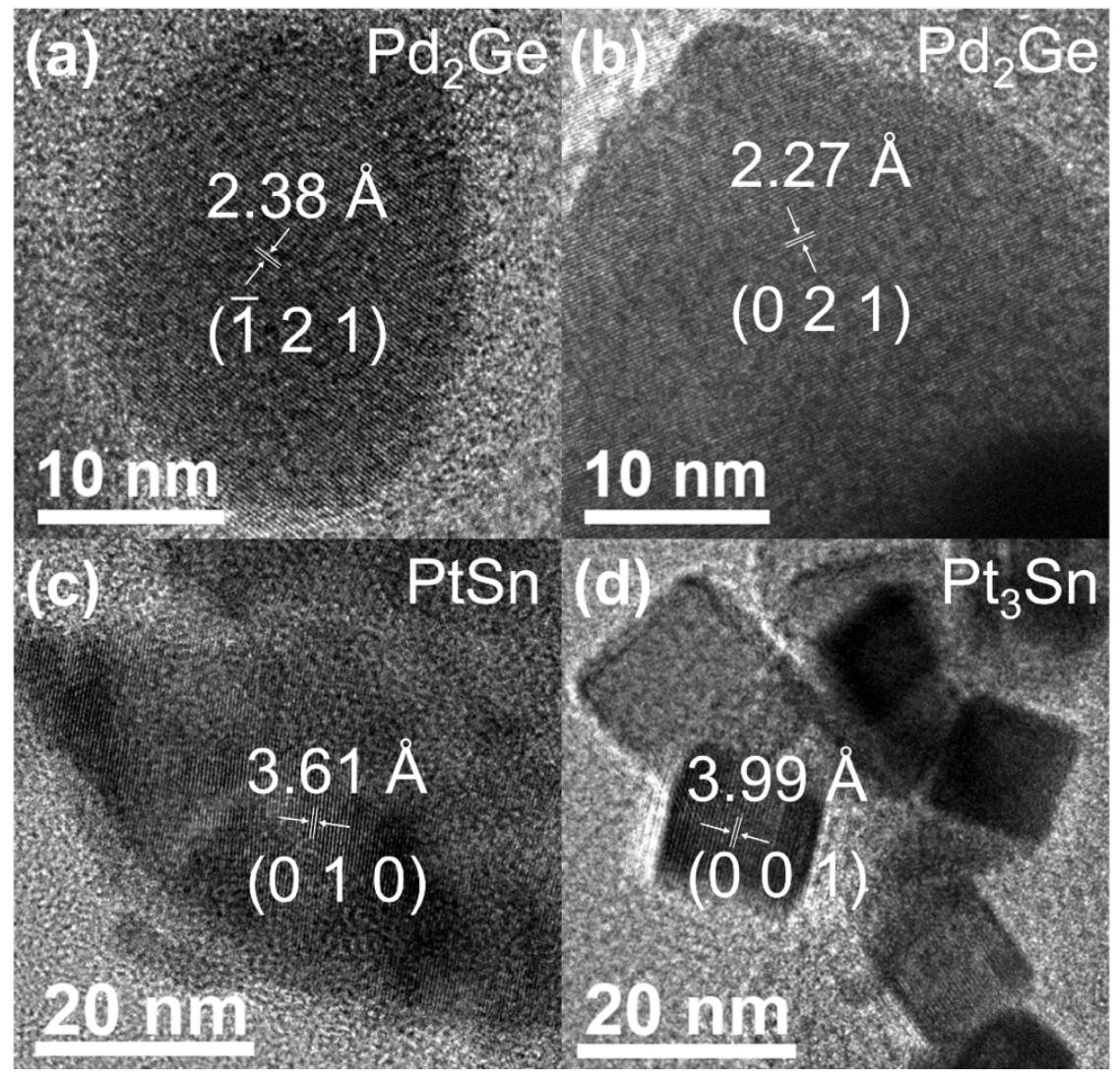

Figure S1. HR TEM of $\mathrm{Pd}_{2} \mathrm{Ge}(\mathrm{a}, \mathrm{b}), \mathrm{PtSn}(\mathrm{c})$, and $\mathrm{Pt}_{3} \mathrm{Sn}(\mathrm{c})$ nanoparticles. In each case, the observed lattice fringes are labeled with their d-spacings and corresponding crystalline lattice planes. 


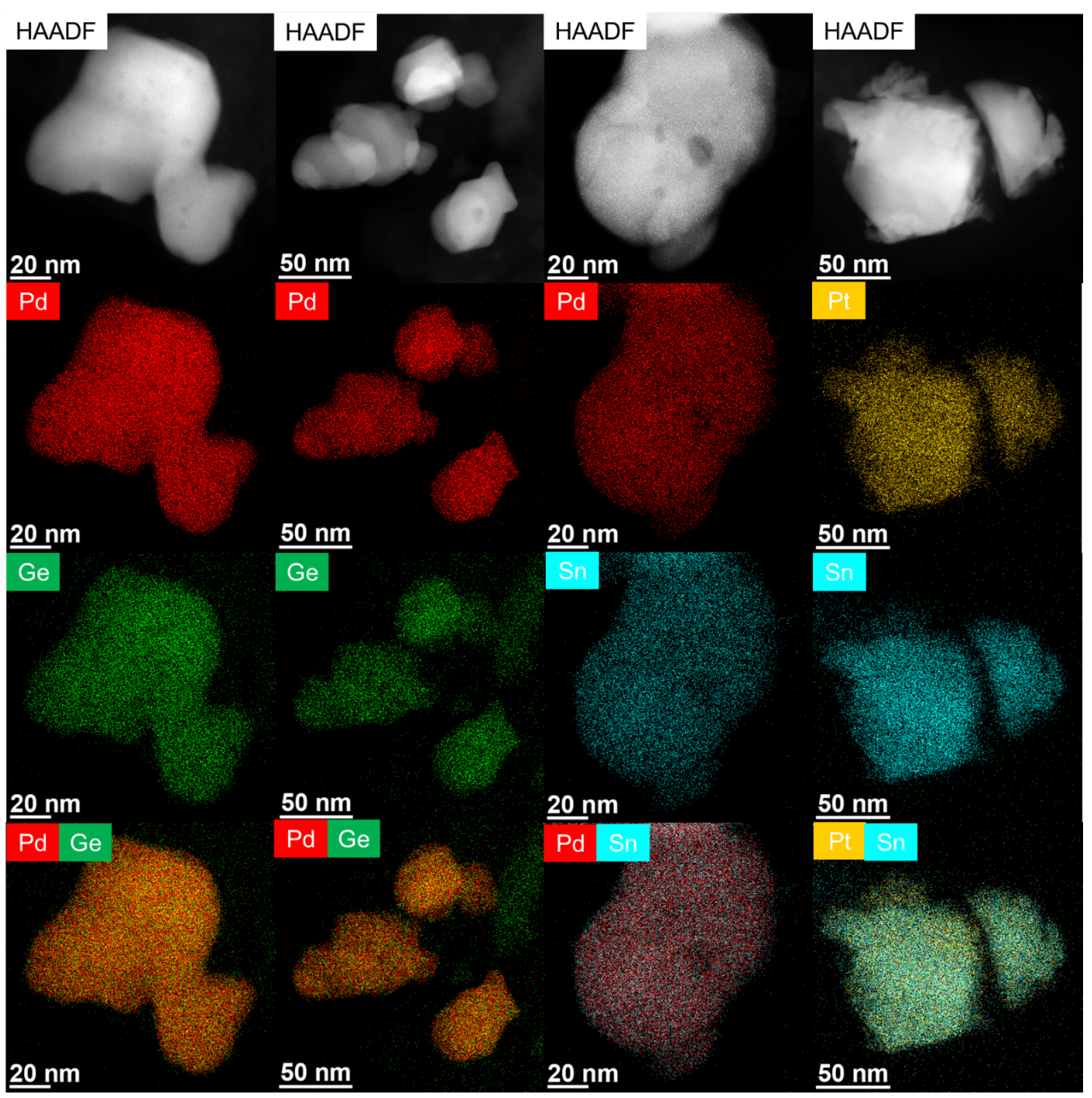

Figure S2. STEM images and the corresponding EDX elemental mapping of the $\operatorname{Pd}_{2} \mathrm{Ge}, \mathrm{Pd}_{2} \mathrm{Sn}$, and PtSn intermetallic nanoparticles. 


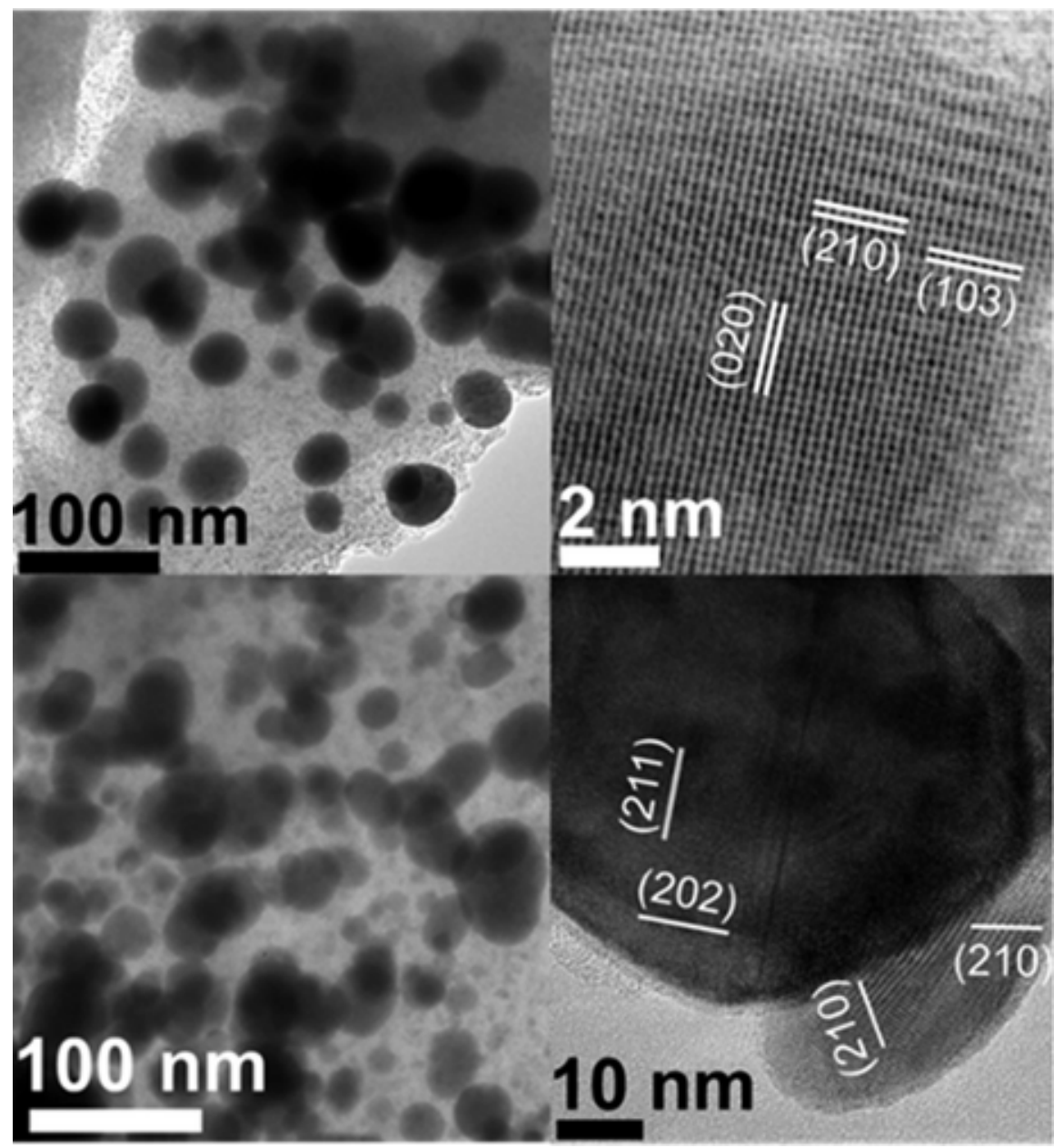

Figure S3. Representative $\mathrm{TEM}$ of $\mathrm{Pd}_{2} \mathrm{Sn}$ nanoparticles produced by the thermolysis of heterobimetallic complex $\mathrm{Cl}_{2} \mathrm{Sn}(\mu \text {-PyS })_{2} \mathrm{PdPPh}_{3}$ at $400{ }^{\circ} \mathrm{C}$. Reprinted with permission from Daniels, C. L.; Knobeloch, M.; Yox, P.; Adamson, M. A. S.; Chen, Y.; Dorn, R. W.; Wu, H.; Zhou, G.; Fan, H.; Rossini, A. J.; Vela, J. Intermetallic Nanocatalysts from Heterobimetallic Group 10-14 Pyridine-2-Thiolate Precursors. Organometallics 2020, 39, 1092-1104. Copyright 2020 American Chemical Society. 


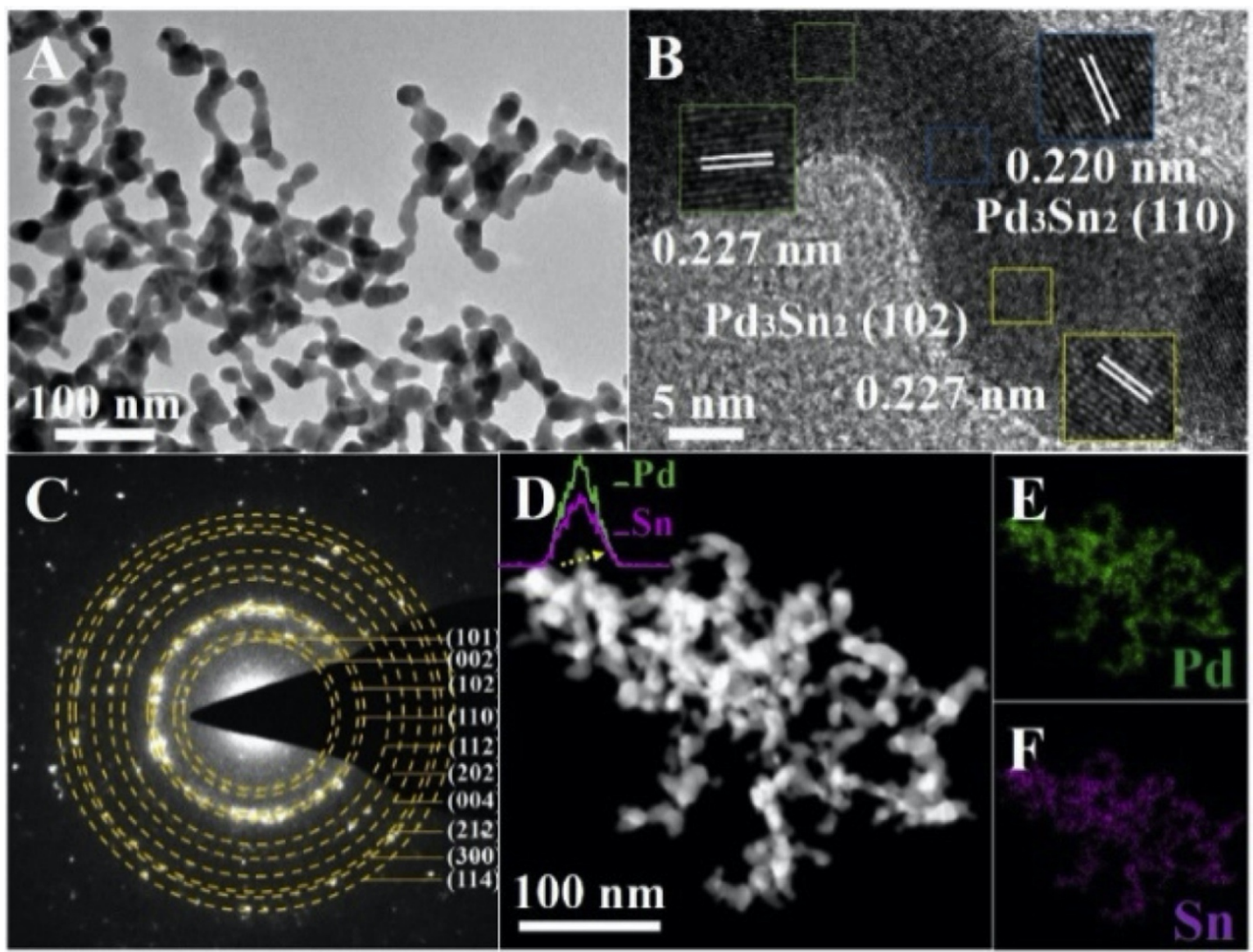

Figure S4. (a) TEM, (b) HR-TEM, and (c) SAED images of $\mathrm{Pd}_{3} \mathrm{Sn}_{2}$ nanonetworks. (d) STEM image of $\mathrm{Pd}_{3} \mathrm{Sn}_{2}$ nanonetworks. Insert: EDAX line-scan curves. The corresponding EDAX maps of (e) Pd and (f) Sn. Reprinted with permission from Li, F.; Xue, Q.; Ma, G.; Li, S.; Hu, M.; Yao, H.; Wang, X. Formic Acid Decomposition-Inhibited Intermetallic $\operatorname{Pd}_{3} \mathrm{Sn}_{2}$ Nanonetworks for Efficient Formic Acid Electrooxidation. J. Power Sources 2020, 450, 227615-1-9. (C) 2019 Elsevier B.V. All rights reserved. 


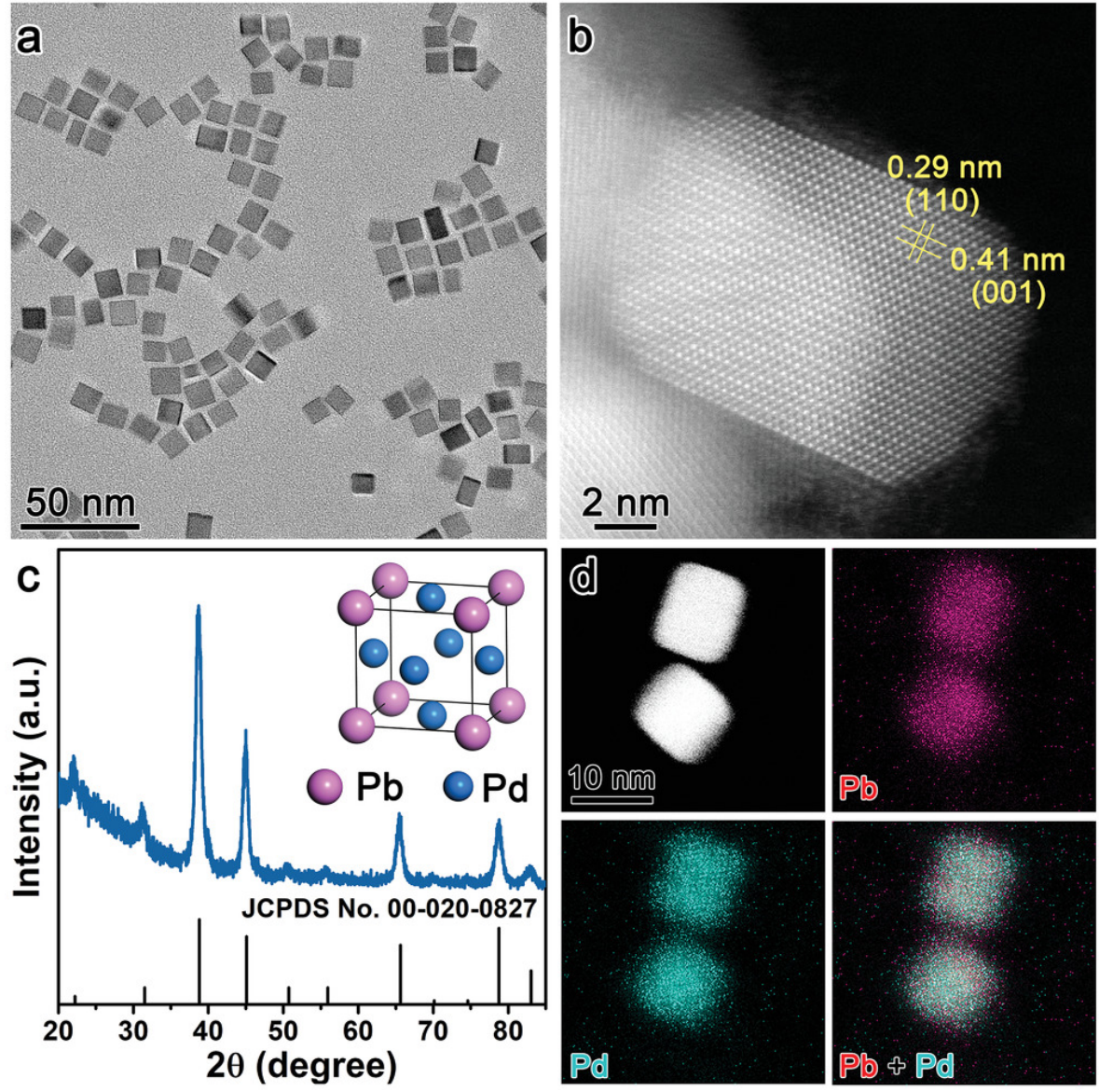

Figure S5. (a) TEM image of the synthesized $\mathrm{Pd}_{3} \mathrm{~Pb}$ intermetallic nanocubes. (b) HAADFSTEM image of a single $\mathrm{Pd}_{3} \mathrm{~Pb}$ intermetallic nanocube. (c) XRD pattern of the $\mathrm{Pd}_{3} \mathrm{~Pb}$ intermetallic nanocubes. The standard diffraction pattern of $\mathrm{Pd} \mathrm{Pd}_{3} \mathrm{~Pb}$ (JCPDS No. 00-020-0827) is used as reference. Inset: Unit cell model of fcc $\mathrm{Pd}_{3} \mathrm{~Pb}$. d) HAADF-STEM and corresponding STEM elemental mapping images of the $\mathrm{Pd}_{3} \mathrm{~Pb}$ intermetallic NCs. Reprinted with permission from Wang, A.-L.; Zhu, L.; Yun, Q.; Han, S.; Zeng, L.; Cao, W.; Meng, X.; Xia, J.; Lu, Q. Bromide Ions Triggered Synthesis of Noble Metal-Based Intermetallic Nanocrystals. Small 2020, 16, 2003782-1-7. (C) 2020 Wiley- VCH GmbH. 
Table S1. Nitro- $\left(\mathrm{PhNO}_{2}, \mathbf{A}\right)$, nitroso-benzene $(\mathrm{PhNO}, \mathbf{I})$, and para-nitrotoluene $\left(p-\mathrm{Me}-\mathrm{C}_{6} \mathrm{H}_{4} \mathrm{NO}_{2}, \mathbf{A}\right)$ reduction data using binary 10-14 intermetallic nanoparticles $v s$. monometallic and alloyed Pd-Sn nanocatalysts. ${ }^{a}$

\begin{tabular}{|c|c|c|c|c|c|c|c|}
\hline \multirow{2}{*}{$\begin{array}{c}\text { Nanocatalyst } \\
\left(\text { Size } \mathrm{nm}^{b}\right)\end{array}$} & \multirow[t]{2}{*}{ Reactant } & \multirow{2}{*}{$\begin{array}{l}\text { Time } \\
\text { (h) }\end{array}$} & \multirow{2}{*}{$\begin{array}{l}\text { Conv. } \\
(\%)\end{array}$} & \multirow{2}{*}{$\begin{array}{c}\mathrm{TON}^{d} \\
\left(\mathrm{TOF}^{e} / \mathrm{h}\right)\end{array}$} & \multicolumn{3}{|c|}{$\operatorname{Selectivity}^{f}(\%)$} \\
\hline & & & & & Azoxy (B) & Azo (C) & Aniline (D) \\
\hline \multirow[t]{3}{*}{ None (control) } & \multirow[t]{3}{*}{$\mathrm{PhNO}_{2}(\mathbf{A})$} & 16 & 9.7 & 0 & 0 & 0 & $>99$ \\
\hline & & 24 & 10.7 & 0 & 0 & 0 & 0 \\
\hline & & 48 & 0 & 0 & 0 & 0 & 0 \\
\hline $\begin{array}{c}\mathrm{Pd}_{2} \mathrm{Ge} \\
(28 \pm 7)\end{array}$ & \multirow[t]{5}{*}{$\mathrm{PhNO}_{2}(\mathbf{A})$} & 4 & 40.1 & $70.6(17.6)$ & 0 & 0 & 0 \\
\hline \multirow[t]{4}{*}{$(28 \pm 7)$} & & 8 & 4.7 & $79.4(9.93)$ & 0 & 0 & 0 \\
\hline & & 16 & 48.9 & $86.5(5.40)$ & $>99$ & 0 & 0 \\
\hline & & 24 & 37.8 & $67.1(2.79)$ & 0 & 0 & 0 \\
\hline & & 48 & 26.6 & $\begin{array}{c}47.6 \\
(0.993)\end{array}$ & $>99$ & 0 & 0 \\
\hline \multirow{4}{*}{$\begin{array}{c}\mathrm{Pd}_{2} \mathrm{Sn}^{8} \\
(49 \pm 11)\end{array}$} & \multirow[t]{4}{*}{$\mathrm{PhNO}_{2}(\mathbf{A})$} & 4 & 44.6 & $171(42.7)$ & 61.9 & 0 & 38.1 \\
\hline & & 8 & 88.6 & $338(42.2)$ & 74.4 & 15.3 & 10.3 \\
\hline & & 16 & 87.7 & $334(20.9)$ & 59.1 & 16.1 & 24.8 \\
\hline & & $16(2)^{i}$ & 74.6 & $284(17.8)$ & 81.1 & 18.9 & 0 \\
\hline
\end{tabular}




\begin{tabular}{|c|c|c|c|c|c|c|c|}
\hline & & 24 & 80.7 & 307 (12.8) & 40.2 & 59.8 & 0 \\
\hline & & 48 & $>99$ & 379 (7.90) & 0 & $>99$ & 0 \\
\hline $\mathrm{Pd}_{3} \mathrm{Sn}_{2^{s}}$ & $\mathrm{PhNO}_{2}(\mathbf{A})$ & 4 & 51.4 & $402(100)$ & $>99$ & 0 & 0 \\
\hline \multirow[t]{4}{*}{$(51 \pm 7)$} & & 8 & 86.5 & $685(85.6)$ & 61.3 & 38.7 & 0 \\
\hline & & 16 & 44.2 & $346(21.7)$ & $>99$ & 0 & 0 \\
\hline & & 24 & 49.5 & 394 (16.4) & 48.5 & 51.5 & 0 \\
\hline & & 48 & 91.8 & $724(15.1)$ & 29.6 & 28.8 & 41.6 \\
\hline $\mathrm{Pd}_{3} \mathrm{~Pb}^{75}$ & $\mathrm{PhNO}_{2}(\mathbf{A})$ & 1 & 93.3 & 194 (194) & 0 & 0 & $>99$ \\
\hline \multirow[t]{6}{*}{$(12 \pm 1)$} & & 2 & $>99$ & $208(104)$ & 0 & 0 & $>99$ \\
\hline & & 4 & $>99$ & $208(52.1)$ & 0 & 0 & $>99$ \\
\hline & & 8 & $>99$ & $208(26.0)$ & 0 & 0 & $>99$ \\
\hline & & 16 & 88.3 & $183(11.5)$ & 0 & 0 & $>99$ \\
\hline & & 24 & 94.7 & $198(8.25)$ & 0 & 0 & $>99$ \\
\hline & & 48 & $>99$ & $208(4.34)$ & 0 & 0 & $>99$ \\
\hline $\mathrm{PtSn}^{8}$ & $\mathrm{PhNO}_{2}(\mathbf{A})$ & 4 & 51.8 & $45.6(11.4)$ & 38.0 & 35.8 & 26.2 \\
\hline \multirow[t]{3}{*}{$(24 \pm 4)$} & & 8 & 68.9 & $60.5(7.57)$ & 43.9 & 56.1 & 0 \\
\hline & & 16 & 51.6 & $45.6(2.85)$ & 81.9 & 18.1 & 0 \\
\hline & & 24 & 66.4 & $57.9(2.41)$ & 61.2 & 38.8 & 0 \\
\hline
\end{tabular}




\begin{tabular}{|c|c|c|c|c|c|c|c|}
\hline & & 48 & 85.9 & $75.4(1.57)$ & 0 & $>99$ & 0 \\
\hline $\mathrm{Pd}^{n}$ & $\mathrm{PhNO}_{2}(\mathbf{A})$ & 1 & 89.1 & $8.09(8.09)$ & 0 & 0 & $>99$ \\
\hline \multirow[t]{6}{*}{$(5 \pm 1)$} & & 2 & 91.1 & $8.27(4.14)$ & 0 & 0 & $>99$ \\
\hline & & 4 & $>99$ & $9.09(2.27)$ & 0 & 0 & $>99$ \\
\hline & & 8 & $>99$ & 9.09 (1.14) & 0 & 0 & $>99$ \\
\hline & & 16 & $>99$ & $\begin{array}{c}9.09 \\
(0.568)\end{array}$ & 0 & 0 & $>99$ \\
\hline & & 24 & $>99$ & $\begin{array}{c}9.09 \\
(0.379)\end{array}$ & 0 & 0 & $>99$ \\
\hline & & 48 & $>99$ & $\begin{array}{c}9.09 \\
(0.189)\end{array}$ & 0 & 0 & $>99$ \\
\hline $\mathrm{Pt}^{n}$ & $\mathrm{PhNO}_{2}(\mathbf{A})$ & 1 & 48.5 & $26.1(26.1)$ & 77.6 & 0 & 22.4 \\
\hline \multirow[t]{6}{*}{$(16 \pm 1)$} & & 2 & 73.9 & 39.4 (19.7) & 53.1 & 3.6 & 43.3 \\
\hline & & 4 & 93.3 & $49.5(12.4)$ & 44.2 & 27.0 & 28.8 \\
\hline & & 8 & $>99$ & $53.2(6.65)$ & 13.9 & 37.2 & 48.9 \\
\hline & & 16 & 69.2 & 36.7 (2.29) & 36.3 & 0 & 63.7 \\
\hline & & 24 & 91.4 & $48.4(2.02)$ & 18.2 & 5.0 & 76.8 \\
\hline & & 48 & 94.8 & $50.5(1.05)$ & 2.5 & 6.9 & 90.6 \\
\hline
\end{tabular}




\begin{tabular}{|c|c|c|c|c|c|c|c|}
\hline$\beta-\mathrm{Sn}^{n}$ & $\mathrm{PhNO}_{2}(\mathbf{A})$ & 1 & 20.0 & $14.9(14.9)$ & 0 & 0 & 0 \\
\hline \multirow[t]{6}{*}{$(55 \pm 6)$} & & 2 & 38.5 & $29.1(14.6)$ & 0 & 0 & 0 \\
\hline & & 4 & 82.4 & $61.2(15.3)$ & $>99$ & 0 & 0 \\
\hline & & 8 & 83.9 & $62.7(7.84)$ & 70.6 & 29.4 & 0 \\
\hline & & 16 & 69.2 & $51.5(3.22)$ & 35.1 & 30.7 & 34.2 \\
\hline & & 24 & 94.4 & $70.2(2.92)$ & 33.7 & 42.6 & 23.7 \\
\hline & & 48 & 95.4 & $70.9(1.48)$ & 0 & 32.7 & 67.3 \\
\hline $\mathrm{Pd}_{0.95} \mathrm{Sn}_{0.05} / \mathrm{C}^{76}$ & $\mathrm{PhNO}_{2}(\mathbf{A})$ & 1 & 93.4 & $18.0(18.0)$ & 0 & 0 & $>99$ \\
\hline \multirow[t]{6}{*}{$(10 \pm 2)$} & & 2 & $>99$ & $19.3(9.67)$ & 0 & 0 & $>99$ \\
\hline & & 4 & $>99$ & $19.3(4.84)$ & 0 & 0 & $>99$ \\
\hline & & 8 & $>99$ & $19.3(2.42)$ & 0 & 0 & $>99$ \\
\hline & & 16 & 71.2 & $\begin{array}{c}13.7 \\
(0.858)\end{array}$ & 0 & 0 & $>99$ \\
\hline & & 24 & $>99$ & $\begin{array}{c}19.3 \\
(0.806)\end{array}$ & 0 & 0 & $>99$ \\
\hline & & 48 & $>99$ & $19.3(4.03)$ & 0 & 0 & $>99$ \\
\hline $\mathrm{Pd}_{0.87} \mathrm{Sn}_{0.13} / \mathrm{C}^{76}$ & $\mathrm{PhNO}_{2}(\mathbf{A})$ & 1 & 92.6 & $15.7(15.7)$ & 0 & 0 & $>99$ \\
\hline$(8 \pm 1)$ & & 2 & $>99$ & $16.9(8.46)$ & 0 & 0 & $>99$ \\
\hline
\end{tabular}




\begin{tabular}{|c|c|c|c|c|c|c|c|}
\hline & & 4 & $>99$ & $16.9(4.23)$ & 0 & 0 & $>99$ \\
\hline & & 8 & $>99$ & $16.9(2.12)$ & 0 & 0 & $>99$ \\
\hline & & 16 & 90.1 & $\begin{array}{c}15.2 \\
(0.952)\end{array}$ & 0 & 0 & $>99$ \\
\hline & & 24 & $>99$ & $\begin{array}{c}16.9 \\
(0.705)\end{array}$ & 0 & 0 & $>99$ \\
\hline & & 48 & 90.6 & $\begin{array}{c}15.4 \\
(0.321)\end{array}$ & 0 & 0 & $>99$ \\
\hline $\mathrm{Au} / \mathrm{TiO}_{2}{ }^{n}$ & $\mathrm{PhNO}_{2}(\mathbf{A})$ & 4 & 93.9 & $1210(302)$ & 24.7 & 0 & 75.3 \\
\hline & & 8 & 95.2 & $1223(153)$ & 0 & 0 & $>99$ \\
\hline & & 16 & $>99$ & $1287(80.4)$ & 0 & 0 & $>99$ \\
\hline None (control) & $p$-Me- & 16 & 0.9 & $0(0)$ & 0 & 0 & 0 \\
\hline & $\mathrm{C}_{6} \mathrm{H}_{4} \mathrm{NO}_{2}(\mathrm{~A})$ & 24 & 24.1 & $0(0)$ & 0 & 0 & 0 \\
\hline & & 48 & 0 & $0(0)$ & 0 & 0 & 0 \\
\hline $\mathrm{Pd}_{2} \mathrm{Ge}_{3}$ & $p$-Me- & 4 & 35.0 & $61.8(15.4)$ & 0 & 0 & 0 \\
\hline$(28 \pm 7)$ & $\mathrm{C}_{6} \mathrm{H}_{4} \mathrm{NO}_{2}(\mathbf{A})$ & 8 & 44.1 & $77.8(9.73)$ & 0 & 0 & 0 \\
\hline & & 16 & 46.5 & $82.1(5.13)$ & 74.6 & 0 & 25.4 \\
\hline & & 24 & 25.9 & $45.7(1.90)$ & 0 & 0 & 0 \\
\hline
\end{tabular}




\begin{tabular}{|c|c|c|c|c|c|c|c|}
\hline & & 48 & 19.5 & $\begin{array}{c}34.4 \\
(0.717)\end{array}$ & 57.7 & 0 & 42.3 \\
\hline $\mathrm{Pd}_{2} \mathrm{Sn}$ & $p$-Me- & 4 & 43.1 & $163(40.9)$ & 87.8 & 12.2 & 0 \\
\hline \multirow[t]{4}{*}{$(49 \pm 11)$} & \multirow[t]{4}{*}{$\mathrm{C}_{6} \mathrm{H}_{4} \mathrm{NO}_{2}(\mathbf{A})$} & 8 & 89.0 & $338(42.2)$ & 68.3 & 25.6 & 6.2 \\
\hline & & 16 & 95.9 & 364 (22.7) & 73.5 & 15.4 & 11.1 \\
\hline & & 24 & 91.2 & $346(14.4)$ & 0 & 36.9 & 63.1 \\
\hline & & 48 & $>99$ & 379 (7.90) & 0 & 20.3 & 79.7 \\
\hline \multirow[t]{5}{*}{$\mathrm{Pd}_{3} \mathrm{Sn}_{2^{s}}$} & \multirow{5}{*}{$\begin{array}{c}p-\mathrm{Me}- \\
\mathrm{C}_{6} \mathrm{H}_{4} \mathrm{NO}_{2}(\mathbf{A})\end{array}$} & 4 & 22.0 & $173(43.3)$ & 0 & 0 & 0 \\
\hline & & 8 & 29.2 & $230(28.7)$ & 0 & 0 & 0 \\
\hline & & 16 & 91.0 & $717(44.8)$ & 82.1 & 17.9 & 0 \\
\hline & & 24 & 97.2 & 765 (31.9) & 43.1 & 28.2 & 28.6 \\
\hline & & 48 & $>99$ & 787 (16.4) & 19.7 & 32.5 & 47.8 \\
\hline \multirow{5}{*}{$(24 \pm 4)$} & \multirow{5}{*}{$\begin{array}{c}p-\mathrm{Me}- \\
\mathrm{C}_{6} \mathrm{H}_{4} \mathrm{NO}_{2}(\mathbf{A})\end{array}$} & 4 & 46.7 & $41.0(10.2)$ & 0 & 0 & $>99$ \\
\hline & & 8 & 75.6 & $66.3(8.29)$ & 35.1 & 48.8 & 16.1 \\
\hline & & 16 & 68.8 & $60.4(3.77)$ & 73.8 & 12.0 & 14.2 \\
\hline & & 24 & 95.8 & $84.0(3.50)$ & 62.8 & 37.2 & 0 \\
\hline & & 48 & $>99$ & $87.7(1.83)$ & 25.3 & 52.4 & 22.3 \\
\hline None & $\mathrm{PhNO}(\mathbf{I})$ & 1 & $>99$ & $\mathrm{~N} / \mathrm{A}$ & $>99$ & 0 & 0 \\
\hline
\end{tabular}




\begin{tabular}{|c|c|c|c|c|c|c|c|}
\hline & & 2 & $>99$ & N/A & $>99$ & 0 & 0 \\
\hline & & 4 & $>99$ & N/A & $>99$ & 0 & 0 \\
\hline & & 8 & $>99$ & N/A & $>99$ & 0 & 0 \\
\hline & & 16 & $>99$ & N/A & 76.8 & 23.2 & 0 \\
\hline & & 24 & $>99$ & $\mathrm{~N} / \mathrm{A}$ & 74.0 & 26.0 & 0 \\
\hline & & 48 & $>99$ & $\mathrm{~N} / \mathrm{A}$ & 80.1 & 19.9 & 0 \\
\hline $\mathrm{Pd}_{2} \mathrm{Sn}^{8}$ & PhNO (I) & 1 & $>99$ & 379 (379) & 53.7 & 46.3 & 0 \\
\hline \multirow[t]{6}{*}{$(49 \pm 11)$} & & 2 & $>99$ & 379 (190) & 38.7 & 61.3 & 0 \\
\hline & & 4 & $>99$ & 379 (94.8) & 27.2 & 72.8 & 0 \\
\hline & & 8 & $>99$ & 379 (47.4) & 0 & $>99$ & 0 \\
\hline & & 16 & $>99$ & 379 (23.7) & 0 & 57.2 & 42.8 \\
\hline & & 24 & $>99$ & 379 (15.8) & 0 & $>99$ & 0 \\
\hline & & 48 & $>99$ & 379 (7.90) & 0 & 100 & 0 \\
\hline \multirow[t]{4}{*}{$\mathrm{Au} / \mathrm{TiO}_{2^{n}}$} & PhNO (I) & 1 & $>99$ & $\begin{array}{l}1287 \\
(1287)\end{array}$ & 10.3 & 41.2 & 48.5 \\
\hline & & 2 & $>99$ & $1287(644)$ & 0 & 37.5 & 62.5 \\
\hline & & 4 & $>99$ & $1287(322)$ & 0 & 15.3 & 84.7 \\
\hline & & 8 & $>99$ & $1287(161)$ & 0 & 10.8 & 89.2 \\
\hline
\end{tabular}




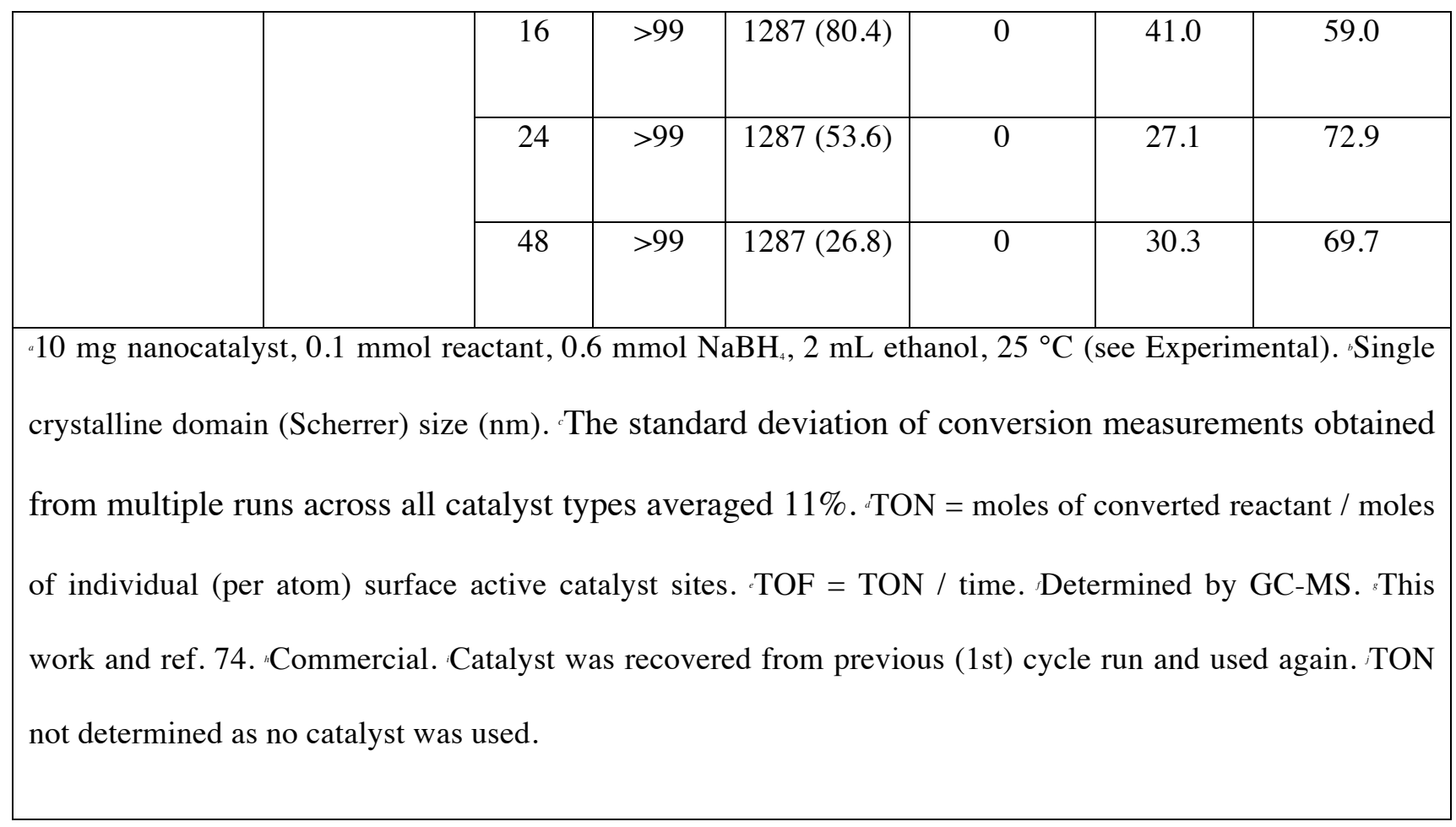

Scheme S2. Catalytic reduction of nitroarenes.<smiles>[R]c1ccc([N+](=O)[O-])cc1</smiles>

A

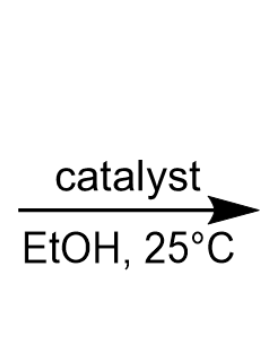

$\mathrm{R}$<smiles>[R]c1ccc(N=Nc2ccc([R])cc2)cc1</smiles><smiles>[R]c1ccc(N)cc1</smiles>

D 


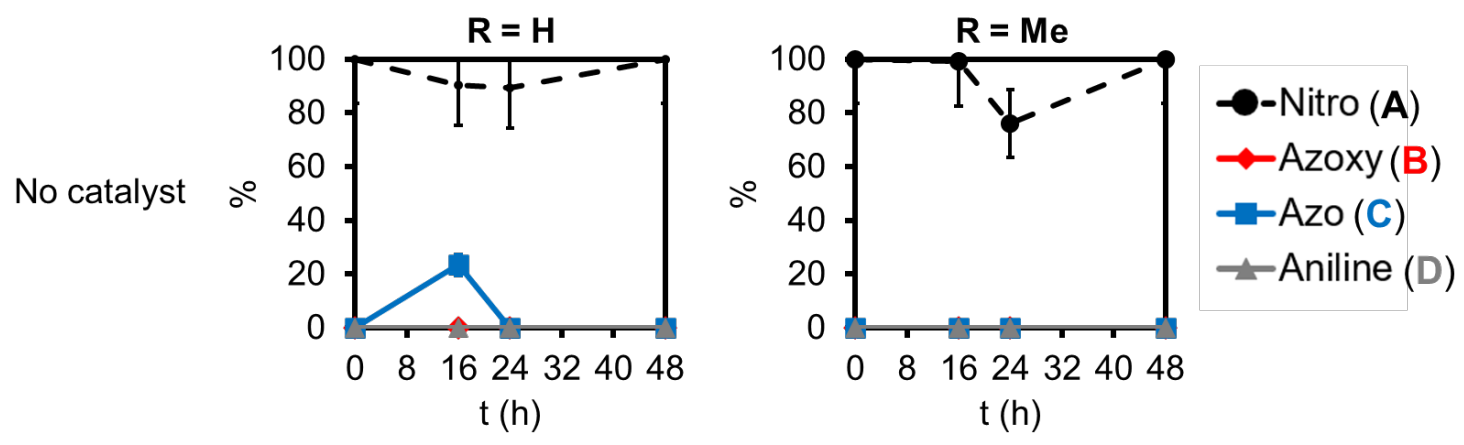

Figure S6. Reduction of nitrobenzene and $p$-nitrotoluene with no catalyst.
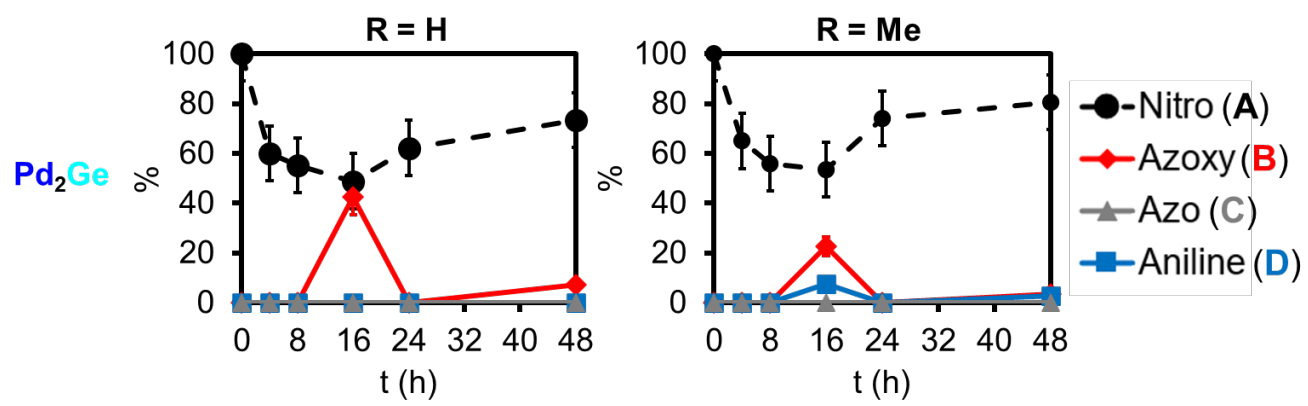

Figure S7. Catalytic reduction of nitrobenzene and $p$-nitrotoluene over $\operatorname{Pd}_{2} \mathrm{Ge}$ nanoparticles.
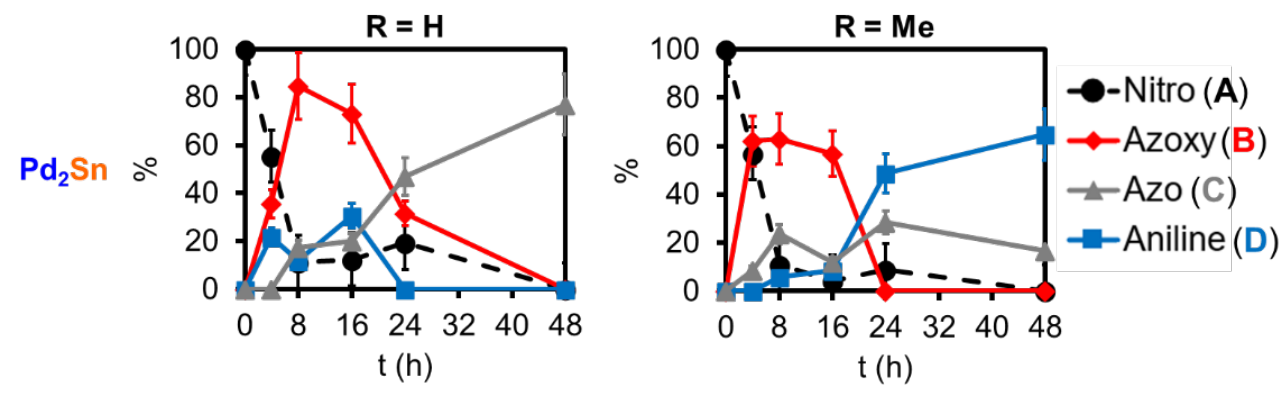

Figure S8. Catalytic reduction of nitrobenzene and $p$-nitrotoluene over $\mathrm{Pd}_{2} \mathrm{Sn}$ nanoparticles . 

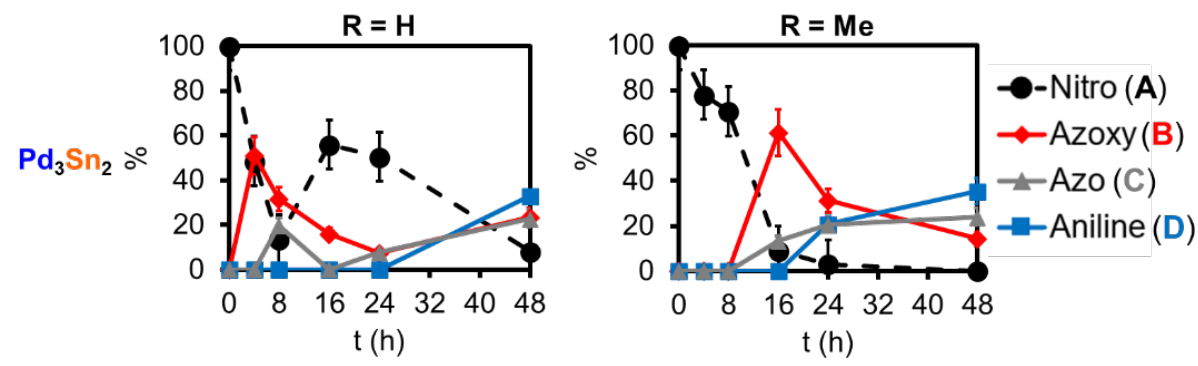

Figure S9. Catalytic reduction of nitrobenzene and $p$-nitrotoluene over $\mathrm{Pd}_{3} \mathrm{Sn}_{2}$ nanoparticles.

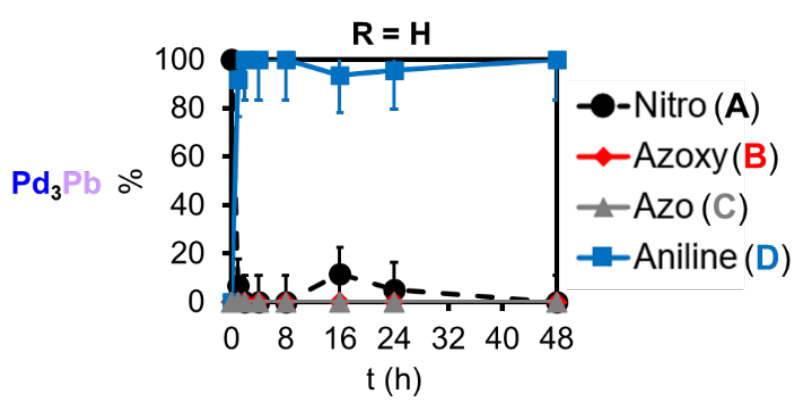

Figure S10. Catalytic reduction of nitrobenzene over $\mathrm{Pd}, \mathrm{Pb}$ nanoparticles.
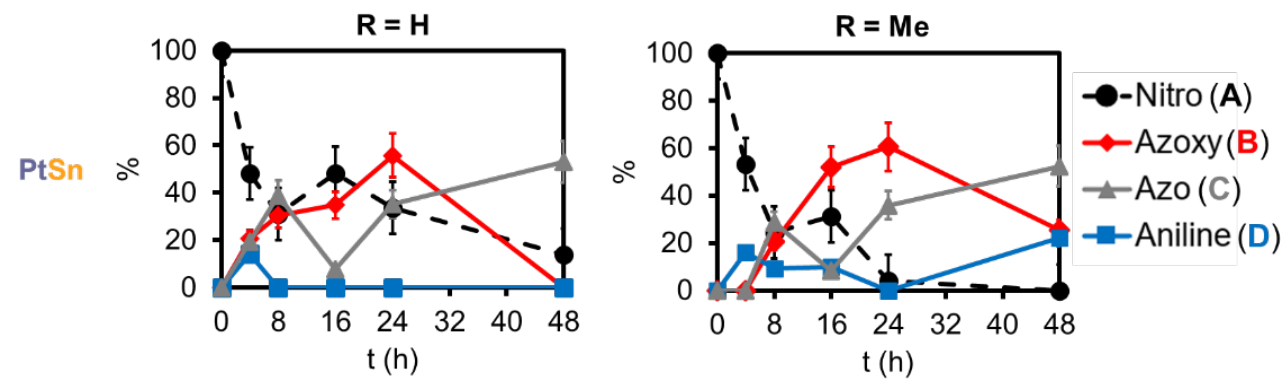

Figure S11. Catalytic reduction of nitrobenzene and $p$-nitrotoluene over PtSn nanoparticles. 

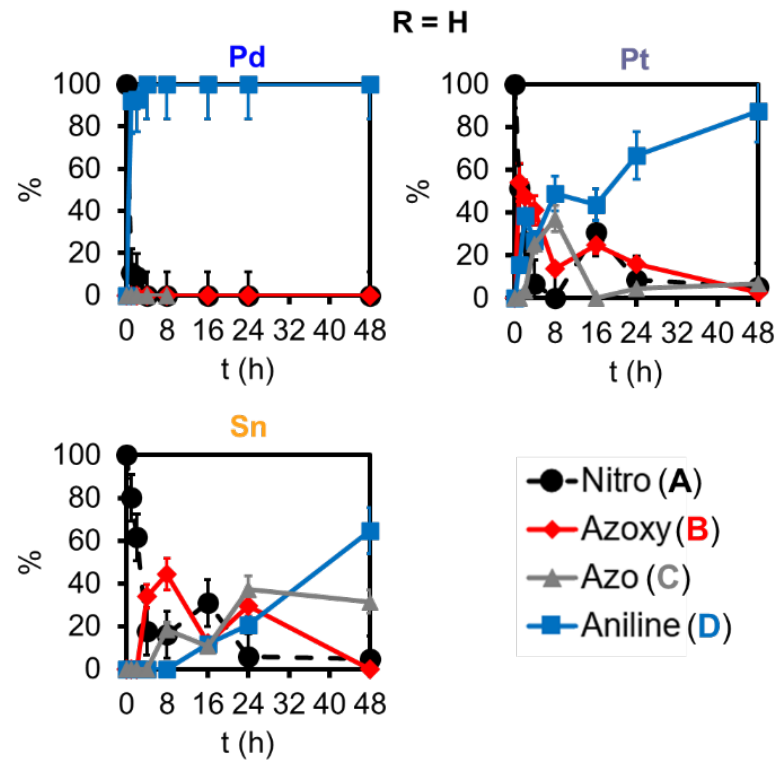

-Nitro (A)

$\multimap A z o x y(B)$

$-\mathrm{Azo}(\mathrm{C})$

- -Aniline (D)

Figure S12. Catalytic reduction of nitrobenzene over Pd, Pt, and Sn nanoparticles. 

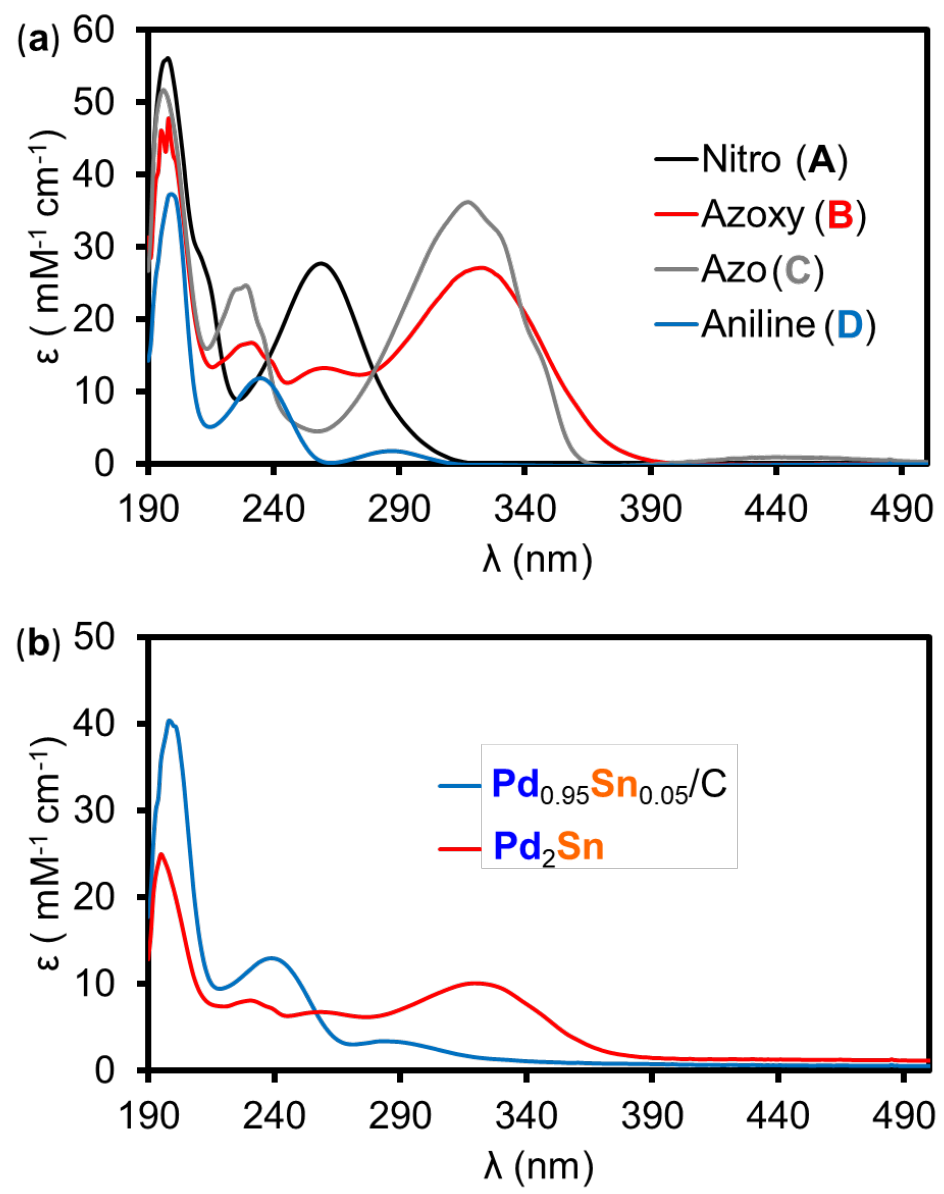

Figure S13. UV-Vis spectra for (a) nitrobenzene, azoxybenzene, azobenzene, and aniline; (b) products from nitrobenzene reduction over $\mathrm{Pd}_{2} \mathrm{Sn}$ nanoparticles (azoxybenzene-selective) vs. $\mathrm{Pd}_{0.95} \mathrm{Sn}_{0.05} / \mathrm{C}$ nanoparticles (aniline-selective). 


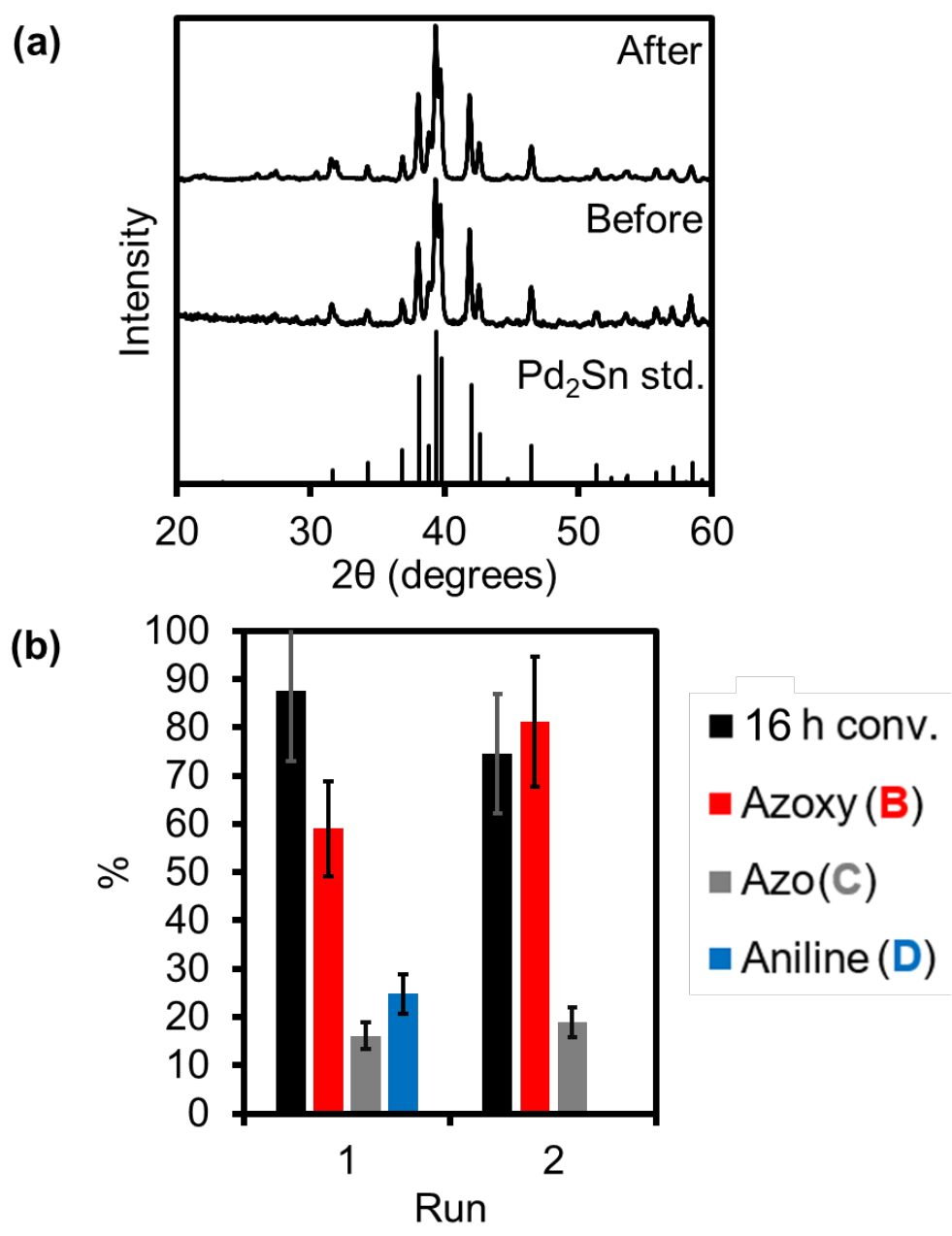

Figure S14. Stability and recyclability study of $\mathrm{Pd}_{2} \mathrm{Sn}$ intermetallic nanoparticle catalyst. (a) XRD patterns of $\mathrm{Pd}_{2} \mathrm{Sn}$ nanoparticles before and after nitrobenzene reduction. (b) $16 \mathrm{~h}$ nitrobenzene reduction data for initial catalyst (1st cycle run) and recovered catalyst (2nd cycle run). 

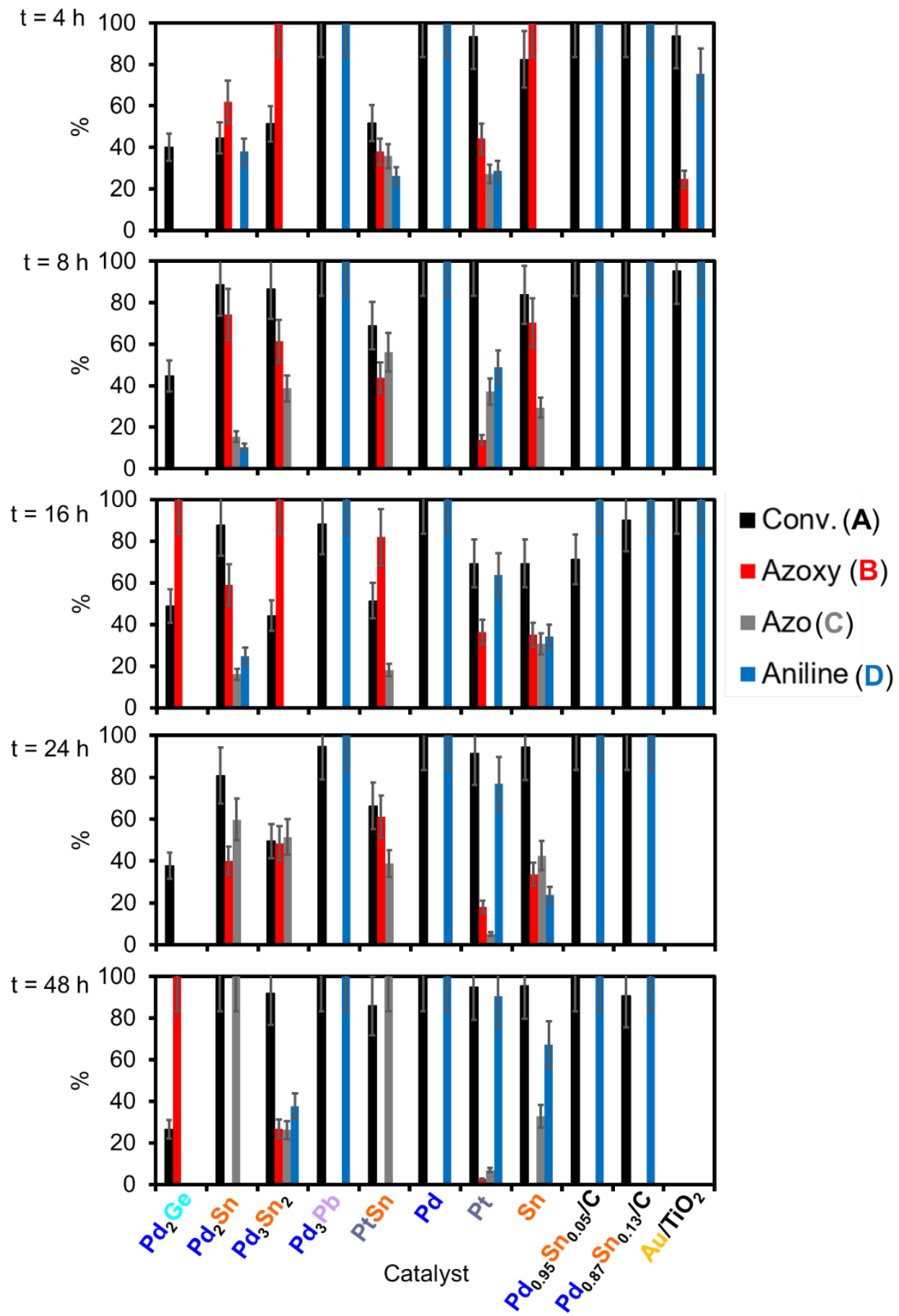

Figure S15. Catalytic reduction of nitrobenzene over binary 10-14 intermetallic nanoparticles, monometallic nanoparticles, and Pd-Sn nanoalloys at different time intervals. 


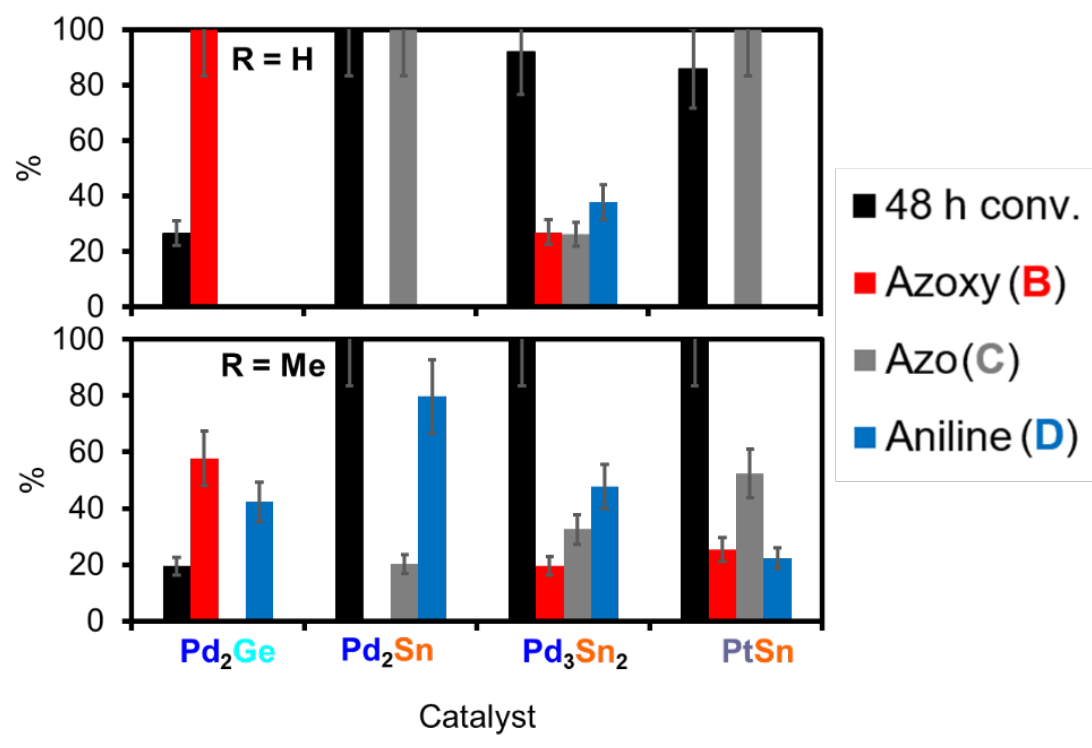

Figure S16. Comparison between the catalytic reduction of nitrobenzene and $p$-nitrotoluene over 10-14 intermetallic nanoparticle catalysts.

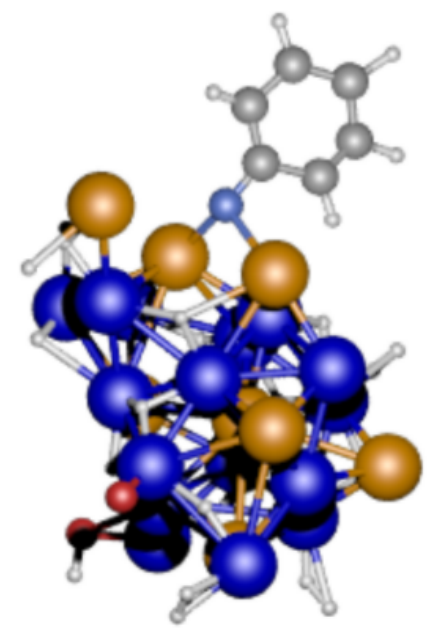

Figure S17. Snapshot of phenylamino radical bonded to two Sn atoms from AIMD simulations. Molecules not bonded with the catalyst are removed for clarity. 
Scheme S3. Mechanistic data observed from DeepMD simulations for the reduction of nitrobenzene over $\mathrm{Pd}_{2} \mathrm{Sn}, \mathrm{Pd}$, or $\mathrm{Sn}$ nanoparticle catalysts.

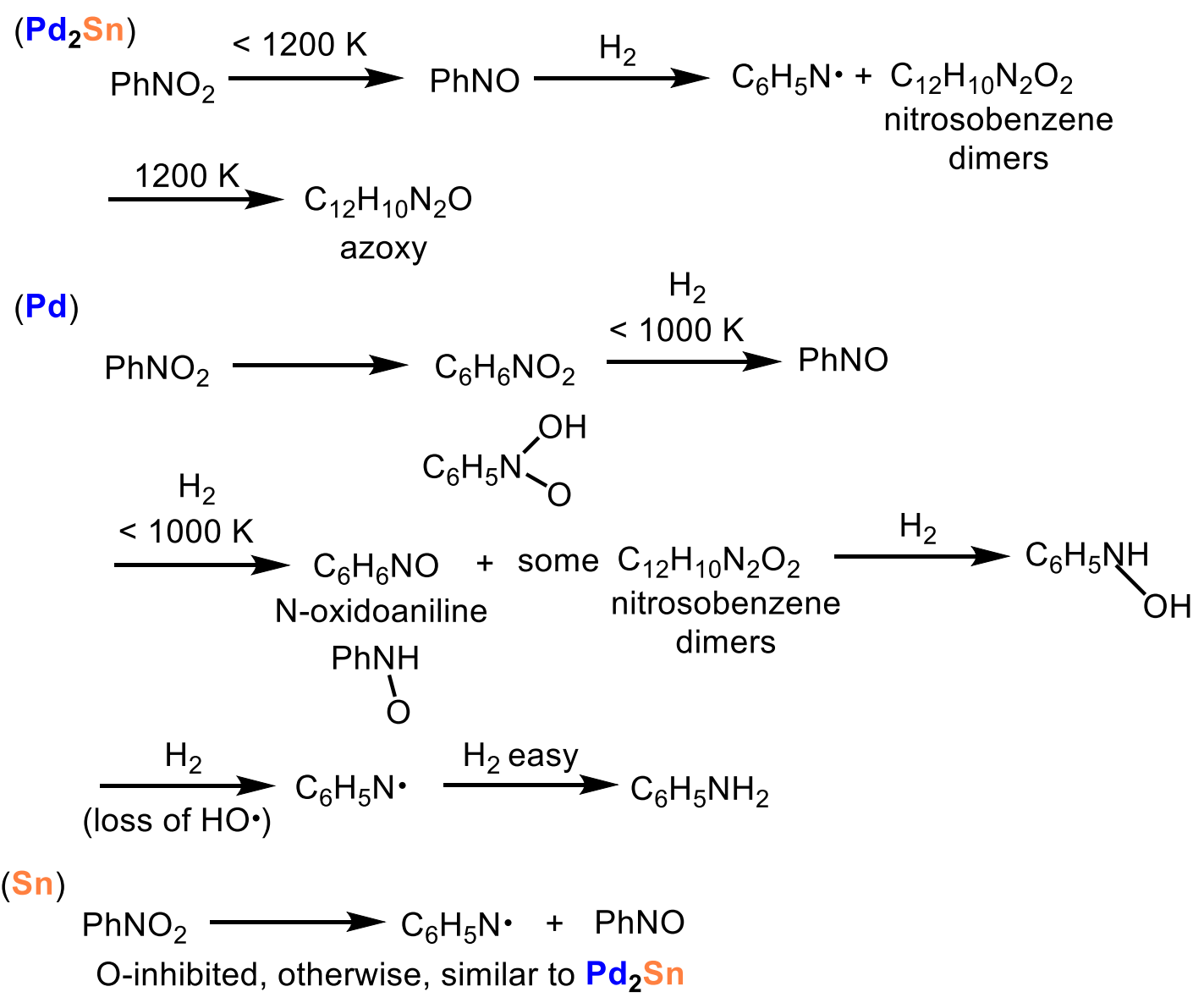




\begin{tabular}{|c|c|c|c|c|c|c|c|c|c|c|c|c|c|c|c|c|c|c|c|c|c|c|}
\hline & tmpr & ntraj & $\mathrm{C} 6 \mathrm{H} 5 \mathrm{NO} 2$ & $\mathrm{C} 2 \mathrm{H} 6 \mathrm{O}$ & $\mathrm{H}$ & но & $\mathrm{H} 2 \mathrm{O}$ & $\mathrm{O}$ & NO2 & $\mathrm{C} 2 \mathrm{H} 5$ & $\mathrm{C} 2 \mathrm{H} 5 \mathrm{O}$ & $\mathrm{C} 2 \mathrm{H} 4 \mathrm{O}$ & C6H5N & $\mathrm{C} 6 \mathrm{H} 5 \mathrm{NO}$ & C6H6 & C6H6N & C6H6NO & C6H6NO2 & $\mathrm{C} 6 \mathrm{H} 7 \mathrm{~N}$ & $\mathrm{C} 6 \mathrm{H} 7 \mathrm{NO}$ & $\mathrm{C} 12 \mathrm{H} 10 \mathrm{~N} 2$ & $\mathrm{C} 12 \mathrm{H} 12 \mathrm{~N} 2$ \\
\hline 0 & $400 \mathrm{~K}$ & 1000 & 1 & 1 & 0.3896 & 0 & 0 & 0 & 0 & 0 & 0 & 0 & 0 & 0 & 0 & 0 & 0 & 0 & 0 & 0 & 0 & 0 \\
\hline 1 & $600 \mathrm{~K}$ & 1000 & 0.9997 & 0.9997 & 0.4566 & 0 & 0 & 0 & 0 & 0 & 0.0003 & 0 & 0 & 0 & 0 & 0 & 0 & 0.0003 & 0 & 0 & 0 & 0 \\
\hline 2 & $800 \mathrm{~K}$ & 1000 & 0.9998 & 0.9998 & 0.4183 & 0 & 0 & 0 & 0 & 0 & 0.0002 & 0 & 0 & 0 & 0 & 0 & 0 & 0.0002 & 0 & 0 & 0 & 0 \\
\hline 3 & $1000 \mathrm{~K}$ & 1000 & 0.9856 & 0.9904 & 0.6522 & 0.0043 & 0 & 0 & 0 & 0 & 0.0096 & 0 & 0 & 0.0043 & 0 & 0 & 0 & 0.0102 & 0 & 0 & 0 & 0 \\
\hline 4 & $1200 \mathrm{~K}$ & 1000 & 0.9433 & 0.9948 & 0.613 & 0.0029 & 0.0151 & 0.0338 & 0 & 0 & 0.0052 & 0 & 0 & 0.0512 & 0 & 0 & 0 & 0.0046 & 0 & 0 & 0 & 0 \\
\hline 5 & $1400 \mathrm{~K}$ & 1000 & 0.8774 & 0.9743 & 0.8338 & 0.0018 & 0.0078 & 0.0668 & 0 & 0 & 0.0249 & 0.0002 & 0 & 0.0648 & 0 & 0 & 0.0116 & 0.0429 & 0 & 0 & 0 & 0 \\
\hline 6 & $1600 \mathrm{~K}$ & 1000 & 0.7996 & 0.9297 & 0.9822 & 0.0614 & 0.0582 & 0.0676 & 0.0133 & 0.0085 & 0.0555 & 0.0001 & 0.0002 & 0.1079 & 0.0087 & 0.019 & 0.0259 & 0.0327 & 0.0003 & 0 & 0 & 0 \\
\hline 7 & $1800 \mathrm{~K}$ & 1000 & 0.6902 & 0.8168 & 1.2987 & 0.1333 & 0.0777 & 0.0806 & 0.0053 & 0.0767 & 0.0758 & 0.0208 & 0.0018 & 0.099 & 0 & 0.0204 & 0.0266 & 0.0947 & 0.0004 & 0.0186 & 0 & 0 \\
\hline 8 & $2000 \mathrm{~K}$ & 1000 & 0.5111 & 0.6629 & 1.3072 & 0.1707 & 0.2492 & 0.0997 & 0.049 & 0.06 & 0.1493 & 0.0854 & 0.0005 & 0.1416 & 0.0979 & 0.027 & 0.0458 & 0.073 & 0.0251 & 0.0191 & 0 & 0 \\
\hline 9 & $2200 \mathrm{~K}$ & 1000 & 0.3293 & 0.4636 & 1.6133 & 0.1302 & 0.5254 & 0.074 & 0.0601 & 0.0842 & 0.1088 & 0.2107 & 0.0119 & 0.1207 & 0.1062 & 0.0245 & 0.0558 & 0.1099 & 0.0438 & 0.0055 & 0 & 0 \\
\hline 10 & $2400 \mathrm{~K}$ & 1000 & 0.2313 & 0.3658 & 1.758 & 0.1411 & 0.687 & 0.0932 & 0.0287 & 0.0735 & 0.1303 & 0.1653 & 0.0109 & 0.1178 & 0.1192 & 0.0083 & 0.0456 & 0.0476 & 0.0288 & 0.0131 & 0 & 0 \\
\hline 11 & $2600 \mathrm{~K}$ & 1000 & 0.1684 & 0.2224 & 2.0826 & 0.2225 & 0.8321 & 0.0809 & 0.0569 & 0.0587 & 0.0812 & 0.151 & 0.004 & 0.0652 & 0.1944 & 0.0315 & 0.0399 & 0.034 & 0.044 & 0.0264 & 0 & 0 \\
\hline 12 & $2800 \mathrm{~K}$ & 1000 & 0.1073 & 0.1623 & 2.1663 & 0.238 & 0.9453 & 0.0757 & 0.0409 & 0.0874 & 0.0999 & 0.1261 & 0.011 & 0.0427 & 0.0997 & 0.0252 & 0.0388 & 0.0393 & 0.0698 & 0.0172 & 0 & 0 \\
\hline 13 & $3000 \mathrm{~K}$ & 1000 & 0.0847 & 0.1057 & 2.5952 & 0.2109 & 1.2598 & 0.0584 & 0.0287 & 0.1405 & 0.0522 & 0.1228 & 0.0014 & 0.0449 & 0.0636 & 0.0095 & 0.0234 & 0.0223 & 0.0101 & 0.0087 & 0 & 0 \\
\hline 14 & & & 0.6234 & 0.692 & 1.2262 & 0.0941 & 0.3327 & 0.0522 & 0.0202 & 0.0421 & 0.0567 & 0.063 & 0.003 & 0.0614 & 0.0493 & 0.0118 & 0.0224 & 0.0365 & 0.0159 & 0.0078 & 0 & 0 \\
\hline
\end{tabular}




\begin{tabular}{|c|c|c|c|c|c|c|c|c|c|c|c|c|c|c|c|c|c|c|c|c|c|c|}
\hline & tmpr & ntraj & C6 65 NO2 & $\mathrm{C} 2 \mathrm{H} 6 \mathrm{O}$ & $\mathrm{H}$ & HO & $\mathrm{H} 2 \mathrm{O}$ & $\mathrm{O}$ & $\mathrm{NO} 2$ & $\mathrm{C} 2 \mathrm{H} 5$ & $\mathrm{C} 2 \mathrm{H} 5 \mathrm{O}$ & $\mathrm{C} 2 \mathrm{H} 4 \mathrm{O}$ & C6H5N & $\mathrm{C} 6 \mathrm{H} 5 \mathrm{NO}$ & $\mathrm{C} 6 \mathrm{H} 6$ & $\mathrm{C} 6 \mathrm{H} 6 \mathrm{~N}$ & $\mathrm{C} 6 \mathrm{H} 6 \mathrm{NO}$ & C6 6 6NO2 & $\mathrm{C} 6 \mathrm{H} 7 \mathrm{~N}$ & C6 $6 \mathrm{H} 7 \mathrm{NO}$ & C12H10N2 & $\mathrm{C} 12 \mathrm{H} 12 \mathrm{~N} 2$ \\
\hline 0 & $400 \mathrm{~K}$ & 1000 & 1 & 1 & 3.5809 & 0 & 0 & 0 & 0 & 0 & 0 & 0 & 0 & 0 & 0 & 0 & 0 & 0 & 0 & 0 & 0 & 0 \\
\hline 1 & $600 \mathrm{~K}$ & 1000 & 1 & 1 & 4.2548 & 0 & 0 & 0 & 0 & 0 & 0 & 0 & 0 & 0 & 0 & 0 & 0 & 0 & 0 & 0 & 0 & 0 \\
\hline 2 & $800 \mathrm{~K}$ & 1000 & 0.9997 & 0.9998 & 5.0546 & 0 & 0 & 0 & 0 & 0 & 0.0002 & 0 & 0 & 0 & 0 & 0 & 0 & 0.0003 & 0 & 0 & 0 & 0 \\
\hline 3 & $1000 \mathrm{~K}$ & 1000 & 0.9791 & 1 & 5.5394 & 0 & 0 & 0 & 0 & 0 & 0 & 0 & 0 & 0 & 0 & 0 & 0 & 0.0208 & 0 & 0 & 0 & 0 \\
\hline 4 & $1200 \mathrm{~K}$ & 1000 & 0.951 & 0.9998 & 5.5956 & 0 & 0 & 0 & 0 & 0 & 0.0003 & 0 & 0 & 0 & 0 & 0 & 0 & 0.0488 & 0 & 0 & 0 & 0 \\
\hline 5 & $1400 \mathrm{~K}$ & 1000 & 0.8662 & 0.9368 & 5.6312 & 0.0002 & 0.0198 & 0 & 0 & 0 & 0.0631 & 0 & 0 & 0.0199 & 0 & 0 & 0.0001 & 0.1073 & 0 & 0 & 0 & 0 \\
\hline 6 & $1600 \mathrm{~K}$ & 1000 & 0.7757 & 0.9137 & 5.7129 & 0.0127 & 0.0585 & 0 & 0.0099 & 0.0024 & 0.0653 & 0.0151 & 0 & 0.0319 & 0.0136 & 0 & 0.0281 & 0.1397 & 0 & 0.0008 & 0 & 0 \\
\hline 7 & $1800 \mathrm{~K}$ & 1000 & 0.6544 & 0.8674 & 5.8342 & 0.0279 & 0.2483 & 0.0002 & 0.0096 & 0.0168 & 0.06 & 0.0177 & 0.0117 & 0.06 & 0.0056 & 0.0102 & 0.031 & 0.0864 & 0.0109 & 0.0343 & 0 & 0 \\
\hline 8 & $2000 \mathrm{~K}$ & 1000 & 0.5437 & 0.7096 & 5.8077 & 0.0276 & 0.4188 & 0.0009 & 0.0081 & 0.0328 & 0.111 & 0.0278 & 0.006 & 0.0882 & 0.0609 & 0.0119 & 0.0901 & 0.1258 & 0.0039 & 0.021 & 0 & 0 \\
\hline 9 & $2200 \mathrm{~K}$ & 1000 & 0.3327 & 0.5241 & 5.7512 & 0.0458 & 0.6493 & 0.0036 & 0.0624 & 0.0558 & 0.1169 & 0.1264 & 0.0031 & 0.0919 & 0.0957 & 0.0261 & 0.052 & 0.0643 & 0.042 & 0.0357 & 0 & 0 \\
\hline 10 & $2400 \mathrm{~K}$ & 1000 & 0.2402 & 0.3428 & 5.8197 & 0.0541 & 0.7292 & 0.0019 & 0.0539 & 0.0472 & 0.1623 & 0.1442 & 0.0161 & 0.0599 & 0.1338 & 0.017 & 0.0348 & 0.0786 & 0.029 & 0.035 & 0 & 0 \\
\hline 11 & $2600 \mathrm{~K}$ & 1000 & 0.1388 & 0.2036 & 5.6992 & 0.0958 & 1.0292 & 0.0032 & 0.0731 & 0.1081 & 0.1186 & 0.1606 & 0.0061 & 0.0331 & 0.0987 & 0.0184 & 0.0366 & 0.0547 & 0.0202 & 0.0221 & 0 & 0 \\
\hline 12 & $2800 \mathrm{~K}$ & 1000 & 0.1159 & 0.1726 & 5.7026 & 0.1274 & 1.1808 & 0.0133 & 0.031 & 0.1042 & 0.0823 & 0.0607 & 0.0155 & 0.0396 & 0.1192 & 0.0125 & 0.0214 & 0.0213 & 0.0086 & 0.0132 & 0 & 0 \\
\hline 13 & $3000 \mathrm{~K}$ & 1000 & 0.0983 & 0.115 & 5.8416 & 0.1149 & 1.1774 & 0.0064 & 0.0228 & 0.1146 & 0.0674 & 0.1125 & 0.0046 & 0.0339 & 0.139 & 0.0154 & 0.0177 & 0.0208 & 0.0197 & 0.0032 & 0 & 0 \\
\hline 14 & & & 0.6211 & 0.6989 & 5.4161 & 0.0362 & 0.3937 & 0.0021 & 0.0193 & 0.0344 & 0.0605 & 0.0475 & 0.0045 & 0.0327 & 0.0476 & 0.008 & 0.0223 & 0.0549 & 0.0096 & 0.0118 & 0 & 0 \\
\hline
\end{tabular}


Table S4. DeepMD simulation results for Sn nanocatalyst starting with 32 nitrobenzene, 32 ethanol, and $384 \mathrm{H} 2$ molecules in a periodic cube with a side length of $3 \mathrm{~nm}$ with a $10 \mathrm{ps}$ time scale.

\begin{tabular}{|c|c|c|c|c|c|c|c|c|c|c|c|c|c|c|c|c|c|c|c|c|c|c|}
\hline & tmpr & ntraj & C6H5NO2 & $\mathrm{C} 2 \mathrm{H} 6 \mathrm{O}$ & $\mathrm{H}$ & $\mathrm{HO}$ & $\mathrm{H} 2 \mathrm{O}$ & $\mathrm{O}$ & $\mathrm{NO} 2$ & $\mathrm{C} 2 \mathrm{H} 5$ & $\mathrm{C} 2 \mathrm{H} 5 \mathrm{O}$ & $\mathrm{C} 2 \mathrm{H} 4 \mathrm{O}$ & C6H5N & C6H5NO & C6H6 & C6H6N & C6H6NO & C6H6NO2 & $\mathrm{C} 6 \mathrm{H} 7 \mathrm{~N}$ & C6H7NO & C12H10N2 & $\mathrm{C} 12 \mathrm{H} 12 \mathrm{~N} 2$ \\
\hline 0 & $400 \mathrm{~K}$ & 1000 & 0.9855 & 1 & 0 & 0 & 0 & 0.0187 & 0 & 0 & 0 & 0 & 0.0042 & 0.0103 & 0 & 0 & 0 & 0 & 0 & 0 & 0 & 0 \\
\hline 1 & $600 \mathrm{~K}$ & 1000 & 0.9026 & 0.9999 & 0 & 0 & 0 & 0.1539 & 0 & 0 & 0.0001 & 0 & 0.0566 & 0.0407 & 0 & 0 & 0 & 0.0001 & 0 & 0 & 0 & 0 \\
\hline 2 & $800 \mathrm{~K}$ & 1000 & 0.9022 & 0.9999 & 0 & 0.0001 & 0 & 0.1633 & 0 & 0 & 0.0001 & 0 & 0.0655 & 0.0323 & 0 & 0 & 0 & 0 & 0 & 0 & 0 & 0 \\
\hline 3 & $1000 \mathrm{~K}$ & 1000 & 0.747 & 0.9999 & 0 & 0 & 0 & 0.4486 & 0 & 0 & 0.0001 & 0 & 0.1957 & 0.0572 & 0 & 0 & 0 & 0.0001 & 0 & 0 & 0 & 0 \\
\hline 4 & $1200 \mathrm{~K}$ & 1000 & 0.6648 & 0.9925 & 0.0001 & 0.002 & 0 & 0.6107 & 0 & 0 & 0.0075 & 0 & 0.283 & 0.0465 & 0 & 0 & 0 & 0.0055 & 0 & 0 & 0 & 0 \\
\hline 5 & $1400 \mathrm{~K}$ & 1000 & 0.4882 & 0.9291 & 0.0005 & 0.0856 & 0 & 0.8143 & 0 & 0 & 0.032 & 0.0389 & 0.3919 & 0.0704 & 0 & 0.0093 & 0 & 0.0266 & 0 & 0 & 0.0008 & 0 \\
\hline 6 & $1600 \mathrm{~K}$ & 1000 & 0.5092 & 0.9577 & 0.0063 & 0.0505 & 0.0055 & 0.8652 & 0 & 0.0066 & 0.0267 & 0.009 & 0.3873 & 0.0523 & 0 & 0.0152 & 0 & 0.0062 & 0 & 0 & 0 & 0 \\
\hline 7 & $1800 \mathrm{~K}$ & 1000 & 0.4873 & 0.9537 & 0.0503 & 0.0298 & 0.0103 & 0.8651 & 0.0001 & 0 & 0.0462 & 0 & 0.3437 & 0.0683 & 0 & 0.0481 & 0.0074 & 0.0219 & 0.0084 & 0 & 0 & 0 \\
\hline 8 & $2000 \mathrm{~K}$ & 1000 & 0.4174 & 0.8077 & 0.0581 & 0.0754 & 0.0602 & 0.9575 & 0.0041 & 0.0383 & 0.0547 & 0.0388 & 0.3359 & 0.0882 & 0.0027 & 0.094 & 0.0192 & 0.0084 & 0.0056 & 0 & 0 & 0 \\
\hline 9 & $2200 \mathrm{~K}$ & 1000 & 0.292 & 0.7117 & 0.2364 & 0.2311 & 0.1129 & 0.9446 & 0.0362 & 0.0742 & 0.061 & 0.0541 & 0.3556 & 0.0849 & 0.0589 & 0.0843 & 0.0061 & 0.0393 & 0.0155 & 0.0014 & 0 & 0 \\
\hline 10 & $2400 \mathrm{~K}$ & 1000 & 0.1826 & 0.523 & 0.2852 & 0.2343 & 0.2226 & 0.9526 & 0.0182 & 0.0417 & 0.198 & 0.0842 & 0.2106 & 0.0706 & 0.1065 & 0.0611 & 0.0126 & 0.0208 & 0.0298 & 0.0037 & 0.0004 & 0 \\
\hline 11 & $2600 \mathrm{~K}$ & 1000 & 0.1303 & 0.4396 & 0.6698 & 0.2676 & 0.3507 & 0.9777 & 0.0272 & 0.0846 & 0.103 & 0.0753 & 0.0792 & 0.0629 & 0.1532 & 0.0765 & 0.0167 & 0.0169 & 0.0394 & 0.0089 & 0 & 0.0003 \\
\hline 12 & $2800 \mathrm{~K}$ & 1000 & 0.1153 & 0.2318 & 0.9233 & 0.4942 & 0.6665 & 0.6564 & 0.0045 & 0.1188 & 0.1049 & 0.1185 & 0.0876 & 0.0486 & 0.1104 & 0.0918 & 0.0124 & 0.0173 & 0.0161 & 0.0022 & 0 & 0.0002 \\
\hline 13 & $3000 \mathrm{~K}$ & 1000 & 0.1132 & 0.1683 & 1.305 & 0.5179 & 0.6819 & 0.6347 & 0.0075 & 0.1334 & 0.0526 & 0.0964 & 0.0695 & 0.0367 & 0.1127 & 0.0447 & 0.006 & 0.0062 & 0.033 & 0.003 & 0.0004 & 0 \\
\hline 14 & & & 0.4956 & 0.7653 & 0.2525 & 0.142 & 0.1508 & 0.6474 & 0.007 & 0.0355 & 0.0491 & 0.0368 & 0.2047 & 0.055 & 0.0389 & 0.0375 & 0.0058 & 0.0121 & 0.0106 & 0.0014 & 0.0001 & 0 \\
\hline
\end{tabular}


Table S5. DeepMD simulation results for $\mathrm{Pd}_{2} \mathrm{Sn}$ nanocatalyst starting with 32 nitrosobenzene and $64 \mathrm{H}_{2}$ molecules in a periodic cube with a side length of $3 \mathrm{~nm}$ with a $10 \mathrm{ps}$ time scale.

\begin{tabular}{|c|c|c|c|c|c|c|c|c|c|c|c|c|c|c|c|c|c|c|c|c|c|c|c|}
\hline & tmpr & ntraj & $\mathrm{C} 6 \mathrm{H} 5 \mathrm{NO}$ & С2 $240 \mathrm{O}$ & $\mathrm{H}$ & $\mathrm{HN}$ & $\mathrm{H} 2 \mathrm{~N}$ & НО & $\mathrm{H} 2 \mathrm{O}$ & $\mathrm{O}$ & NO & $\mathrm{C} 6 \mathrm{H} 5$ & $\mathrm{C} 6 \mathrm{H} 5 \mathrm{~N}$ & C6H6 & $\mathrm{C} 6 \mathrm{H} 6 \mathrm{~N}$ & C6H6NO & C6H6NO2 & $\mathrm{C} 6 \mathrm{H} 7 \mathrm{~N}$ & $\mathrm{C} 6 \mathrm{H} 7 \mathrm{NO}$ & C6 $6 \mathrm{H} 7 \mathrm{NO} 2$ & $\mathrm{C} 12 \mathrm{H} 10 \mathrm{~N} 2$ & $\mathrm{C} 12 \mathrm{H} 10 \mathrm{~N} 2 \mathrm{O}$ & $\mathrm{C} 12 \mathrm{H} 10 \mathrm{~N} 2 \mathrm{O} 2$ \\
\hline 0 & $400 \mathrm{~K}$ & 1000 & 0.8485 & 0 & 0 & 0 & 0 & 0 & 0 & 0 & 0 & 0 & 0 & 0 & 0 & 0 & 0 & 0 & 0 & 0 & 0 & 0 & 0.0757 \\
\hline 1 & $600 \mathrm{~K}$ & 1000 & 0.8233 & 0 & 0 & 0 & 0 & 0 & 0 & 0 & 0 & 0 & 0.0027 & 0 & 0 & 0 & 0 & 0 & 0 & 0 & 0 & 0 & 0.0856 \\
\hline 2 & $800 \mathrm{~K}$ & 1000 & 0.8879 & 0 & 0 & 0 & 0 & 0 & 0 & 0.0275 & 0 & 0 & 0.0275 & 0 & 0 & 0 & 0 & 0 & 0 & 0 & 0 & 0 & 0.0423 \\
\hline 3 & $1000 \mathrm{~K}$ & 1000 & 0.9019 & 0 & 0 & 0 & 0 & 0 & 0 & 0.0119 & 0.0001 & 0.0001 & 0.0136 & 0 & 0 & 0 & 0 & 0 & 0 & 0 & 0 & 0 & 0.0414 \\
\hline 4 & $1200 \mathrm{~K}$ & 1000 & 0.9235 & 0 & 0 & 0 & 0 & 0 & 0 & 0.0246 & 0.0008 & 0.0008 & 0.0178 & 0 & 0 & 0 & 0 & 0 & 0 & 0 & 0 & 0.0068 & 0.0221 \\
\hline 5 & $1400 \mathrm{~K}$ & 1000 & 0.7191 & 0 & 0.0001 & 0 & 0 & 0 & 0 & 0.017 & 0.1802 & 0.1813 & 0.0231 & 0.0002 & 0 & 0 & 0 & 0 & 0 & 0 & 0 & 0.0191 & 0.0057 \\
\hline 6 & $1600 \mathrm{~K}$ & 1000 & 0.6594 & 0 & 0.0213 & 0 & 0 & 0 & 0 & 0.0231 & 0.261 & 0.1764 & 0.0567 & 0.0131 & 0.0006 & 0.0001 & 0 & 0 & 0 & 0 & 0 & 0.0004 & 0.0018 \\
\hline 7 & $1800 \mathrm{~K}$ & 1000 & 0.4079 & 0 & 0.0617 & 0 & 0 & 0.0003 & 0 & 0.127 & 0.4088 & 0.2965 & 0.1046 & 0.0501 & 0.0001 & 0 & 0 & 0 & 0 & 0 & 0 & 0 & 0.0022 \\
\hline 8 & $2000 \mathrm{~K}$ & 1000 & 0.3103 & 0 & 0.0474 & 0 & 0 & 0.0277 & 0 & 0.06 & 0.5249 & 0.4063 & $\begin{array}{l}0.0873 \\
\end{array}$ & 0.05 & 0.0055 & 0.0002 & 0 & 0 & 0 & 0 & 0 & 0 & 0.0074 \\
\hline 9 & $2200 \mathrm{~K}$ & 1000 & 0.2231 & 0 & 0.0536 & 0 & 0 & 0 & 0 & 0.0509 & 0.64 & 0.4679 & 0.0254 & 0.1213 & 0 & 0.0003 & 0 & 0 & 0 & 0 & 0 & 0 & 0.0016 \\
\hline 10 & $2400 \mathrm{~K}$ & 1000 & 0.1769 & 0 & 0.0261 & 0 & 0 & 0.0225 & 0.0004 & 0.0711 & 0.6673 & 0.4698 & 0.0233 & 0.1215 & 0 & 0.0043 & 0 & 0 & 0 & 0 & 0 & 0.0001 & 0.0011 \\
\hline 11 & $2600 \mathrm{~K}$ & 1000 & 0.1383 & 0 & 0.0946 & 0.001 & 0 & 0.0268 & 0.0102 & 0.1459 & 0.5164 & 0.3073 & 0.0432 & 0.1326 & 0.0039 & 0.016 & 0 & 0.0016 & 0.0058 & 0 & 0 & 0 & 0.0014 \\
\hline 12 & $2800 \mathrm{~K}$ & 1000 & 0.0843 & 0 & 0.2102 & 0.0216 & 0.0003 & 0.0399 & 0.028 & 0.1779 & 0.5605 & 0.2198 & 0.0377 & 0.1037 & 0.0027 & 0.0011 & 0 & 0.0016 & 0 & 0 & 0 & 0.0005 & 0.0009 \\
\hline 13 & $3000 \mathrm{~K}$ & 1000 & 0.0725 & 0 & 0.2414 & 0.0215 & 0.0019 & 0.0322 & 0.0034 & 0.0316 & 0.5475 & 0.2384 & 0.015 & 0.1223 & 0.0068 & 0.003 & 0.0001 & 0.0028 & 0 & 0 & 0 & 0 & 0.0015 \\
\hline 14 & & & 0.5126 & 0 & 0.054 & 0.0031 & 0.0002 & 0.0107 & 0.003 & 0.0549 & 0.3077 & 0.1975 & 0.0341 & 0.051 & 0.0014 & 0.0018 & 0 & 0.0004 & 0.0004 & 0 & 0 & 0.0019 & 0.0208 \\
\hline
\end{tabular}




\begin{tabular}{|c|c|c|c|c|c|c|c|c|c|c|c|c|c|c|c|c|c|c|c|}
\hline & tmpr & ntraj & $\mathrm{C} 12 \mathrm{H} 10 \mathrm{~N} 2 \mathrm{O}$ & $\mathrm{C} 2 \mathrm{H} 6 \mathrm{O}$ & $\mathrm{H}$ & $\mathrm{HN}$ & $\mathrm{H} 2 \mathrm{~N}$ & HO & $\mathrm{H} 2 \mathrm{O}$ & $\mathrm{O}$ & NO & $\mathrm{C} 6 \mathrm{H} 5$ & $\mathrm{C} 6 \mathrm{H} 5 \mathrm{~N}$ & $\mathrm{C} 6 \mathrm{H} 6$ & $\mathrm{C} 6 \mathrm{H} 6 \mathrm{~N}$ & $\mathrm{C} 6 \mathrm{H} 6 \mathrm{NO} 2$ & $\mathrm{C} 6 \mathrm{H} 7 \mathrm{~N}$ & $\mathrm{C} 6 \mathrm{H} 7 \mathrm{NO}$ & $\mathrm{C} 12 \mathrm{H} 10 \mathrm{~N} 2$ \\
\hline 0 & $400 \mathrm{~K}$ & 1000 & 1 & 0 & 0 & 0 & 0 & 0 & 0 & 0 & 0 & 0 & 0 & 0 & 0 & 0 & 0 & 0 & 0 \\
\hline 1 & $600 \mathrm{~K}$ & 1000 & 1 & 0 & 0.0263 & 0 & 0 & 0 & 0 & 0 & 0 & 0 & 0 & 0 & 0 & 0 & 0 & 0 & 0 \\
\hline 2 & $800 \mathrm{~K}$ & 1000 & 1 & 0 & 0.0001 & 0 & 0 & 0 & 0 & 0 & 0 & 0 & 0 & 0 & 0 & 0 & 0 & 0 & 0 \\
\hline 3 & $1000 \mathrm{~K}$ & 1000 & 0.9967 & 0 & 0.0012 & 0 & 0 & 0 & 0 & 0.0022 & 0 & 0.0007 & 0.0003 & 0 & 0 & 0 & 0 & 0 & 0.0022 \\
\hline 4 & $1200 \mathrm{~K}$ & 1000 & 0.9432 & 0 & 0.0406 & 0 & 0 & 0 & 0 & 0.0338 & 0 & 0.0027 & 0.0073 & 0 & 0 & 0 & 0 & 0 & 0.0338 \\
\hline 5 & $1400 \mathrm{~K}$ & 1000 & 0.9407 & 0 & 0.0443 & 0 & 0 & 0 & 0 & 0 & 0.0084 & 0.0083 & 0.0317 & 0 & 0.0066 & 0 & 0 & 0 & 0.0007 \\
\hline 6 & $1600 \mathrm{~K}$ & 1000 & 0.7158 & 0 & 0.115 & 0.0004 & 0 & 0 & 0 & 0.034 & 0.0193 & 0.1136 & 0.0581 & 0.0845 & 0.0006 & 0 & 0 & 0 & 0.0158 \\
\hline 7 & $1800 \mathrm{~K}$ & 1000 & 0.4833 & 0 & 0.0801 & 0 & 0 & 0 & 0 & 0.0267 & 0.0184 & 0.1856 & 0.0866 & 0.1021 & 0.0482 & 0 & 0 & 0 & 0.0041 \\
\hline 8 & $2000 \mathrm{~K}$ & 1000 & 0.3347 & 0 & 0.1983 & 0 & 0.0043 & 0.0178 & 0.0073 & 0.0406 & 0.1464 & 0.1941 & 0.0905 & 0.1595 & 0.0527 & 0 & 0.0047 & 0.0001 & 0.0161 \\
\hline 9 & $2200 \mathrm{~K}$ & 1000 & 0.1935 & 0 & 0.2004 & 0 & 0 & 0.0361 & 0 & 0.0308 & 0.1298 & 0.1493 & 0.1652 & 0.2003 & 0.1573 & 0 & 0.0208 & 0.0008 & 0.0105 \\
\hline 10 & $2400 \mathrm{~K}$ & 1000 & 0.1143 & 0 & 0.2622 & 0.0002 & 0.0007 & 0.0488 & 0.0431 & 0.0782 & 0.2099 & 0.1787 & 0.1514 & 0.3052 & 0.106 & 0 & 0.0539 & 0.0013 & 0.0772 \\
\hline 11 & $2600 \mathrm{~K}$ & 1000 & 0.1119 & 0 & 0.1985 & 0.0062 & 0.0012 & 0.0233 & 0.0438 & 0.0314 & 0.2587 & 0.2093 & 0.1394 & 0.3477 & 0.0752 & 0 & 0.0278 & 0.0004 & 0.017 \\
\hline 12 & $2800 \mathrm{~K}$ & 1000 & 0.0704 & 0 & 0.3652 & 0.013 & 0.0088 & 0.0315 & 0.0969 & 0.0644 & 0.2268 & 0.2047 & 0.1207 & 0.1643 & 0.0659 & 0.0072 & 0.0152 & 0.0002 & 0.0062 \\
\hline 13 & $3000 \mathrm{~K}$ & 1000 & 0.0701 & 0 & 0.4085 & 0.0053 & 0.0205 & 0.0564 & 0.0928 & 0.0546 & 0.1762 & 0.0919 & 0.0794 & 0.1212 & 0.0411 & 0 & 0.0156 & 0.0034 & 0.0006 \\
\hline 14 & & & 0.5696 & 0 & 0.1386 & 0.0018 & 0.0025 & 0.0153 & 0.0203 & 0.0283 & 0.0853 & 0.0956 & 0.0665 & 0.1061 & 0.0395 & 0.0005 & 0.0099 & 0.0004 & 0.0132 \\
\hline
\end{tabular}




\begin{tabular}{|c|c|c|c|c|c|c|c|c|c|c|c|c|c|c|c|c|}
\hline & tmpr & ntraj & $\mathrm{C} 6 \mathrm{H} 6 \mathrm{NO} 2$ & $\mathrm{H}$ & $\mathrm{HO}$ & $\mathrm{H} 2 \mathrm{O}$ & $\mathrm{O}$ & C6H5N & $\mathrm{C} 6 \mathrm{H} 5 \mathrm{NO}$ & $\mathrm{C} 6 \mathrm{H} 5 \mathrm{NO} 2$ & С6H6 & C6H6N & $\mathrm{C} 6 \mathrm{H} 7 \mathrm{~N}$ & $\mathrm{C} 6 \mathrm{H} 7 \mathrm{NO}$ & $\mathrm{C} 6 \mathrm{H} 7 \mathrm{NO} 2$ & $\mathrm{C} 12 \mathrm{H} 10 \mathrm{~N} 2 \mathrm{O}$ \\
\hline 0 & $400 \mathrm{~K}$ & 1000 & 0.9343 & 1.7572 & 0 & 0 & 0 & 0 & 0 & 0.0328 & 0 & 0 & 0 & 0 & 0.0328 & 0 \\
\hline 1 & $600 \mathrm{~K}$ & 1000 & 0.9261 & 2.1499 & 0 & 0 & 0 & 0 & 0 & 0.037 & 0 & 0 & 0 & 0 & 0.037 & 0 \\
\hline 2 & $800 \mathrm{~K}$ & 1000 & 0.8354 & 2.292 & 0 & 0 & 0 & 0 & 0 & 0.0823 & 0 & 0 & 0 & 0 & 0.0823 & 0 \\
\hline 3 & $1000 \mathrm{~K}$ & 1000 & 0.5562 & 1.881 & 0.035 & 0.1323 & 0 & 0 & 0.1147 & 0.2103 & 0 & 0 & 0 & 0 & 0.0503 & 0 \\
\hline 4 & $1200 \mathrm{~K}$ & 1000 & 0.47 & 1.9748 & 0.0162 & 0.2136 & 0 & 0.0088 & 0.1554 & 0.2767 & 0.006 & 0.0014 & 0 & 0 & 0.0326 & 0 \\
\hline 5 & $1400 \mathrm{~K}$ & 1000 & 0.3686 & 2.0125 & 0.0563 & 0.1858 & 0.0062 & 0.0026 & 0.1153 & 0.3659 & 0 & 0.011 & 0.0152 & 0.0308 & 0.0421 & 0 \\
\hline 6 & $1600 \mathrm{~K}$ & 1000 & 0.3064 & 1.9669 & 0.0691 & 0.2868 & 0 & 0.0027 & 0.1454 & 0.297 & 0.0507 & 0.0027 & 0.0165 & 0.0013 & 0.0152 & 0 \\
\hline 7 & $1800 \mathrm{~K}$ & 1000 & 0.2056 & 1.7347 & 0.0889 & 0.4584 & 0.0019 & 0.0145 & 0.1623 & 0.3122 & 0.0725 & 0.0107 & 0.0214 & 0.0265 & 0.0108 & 0 \\
\hline 8 & $2000 \mathrm{~K}$ & 1000 & 0.1904 & 1.7565 & 0.0568 & 0.5648 & 0.0032 & 0.0019 & 0.284 & 0.1972 & 0.0633 & 0.0167 & 0.02 & 0.0149 & 0.0026 & 0.0044 \\
\hline 9 & & & 0.5326 & 1.9473 & 0.0358 & 0.2046 & 0.0013 & 0.0034 & 0.1086 & 0.2013 & 0.0214 & 0.0047 & 0.0081 & 0.0082 & 0.034 & 0.0005 \\
\hline
\end{tabular}




\begin{tabular}{|c|c|c|c|c|c|c|c|c|c|c|c|c|c|}
\hline & tmpr & ntraj & $\mathrm{C} 6 \mathrm{H} 5 \mathrm{NO}$ & $\mathrm{H}$ & $\mathrm{HO}$ & $\mathrm{H} 2 \mathrm{O}$ & $\mathrm{O}$ & $\mathrm{C} 6 \mathrm{H} 5$ & C6H6 & C6H6NO & $\mathrm{C} 6 \mathrm{H} 7 \mathrm{~N}$ & $\mathrm{C} 12 \mathrm{H} 10 \mathrm{~N} 2 \mathrm{O}$ & $\mathrm{C} 12 \mathrm{H} 10 \mathrm{~N} 2 \mathrm{O} 2$ \\
\hline 0 & $400 \mathrm{~K}$ & 1000 & 0.8932 & 1.5514 & 0 & 0 & 0 & 0 & 0 & 0 & 0 & 0 & 0.0534 \\
\hline 1 & $600 \mathrm{~K}$ & 1000 & 0.8373 & 1.9102 & 0 & 0 & 0 & 0 & 0 & 0.0519 & 0 & 0 & 0.0554 \\
\hline 2 & $800 \mathrm{~K}$ & 1000 & 0.77 & 2.0873 & 0 & 0 & 0 & 0.0173 & 0 & 0.0695 & 0 & 0 & 0.0664 \\
\hline 3 & $1000 \mathrm{~K}$ & 1000 & 0.776 & 2.1017 & 0.0218 & 0 & 0 & 0 & 0 & 0.0702 & 0.0001 & 0 & 0.0483 \\
\hline 4 & $1200 \mathrm{~K}$ & 1000 & 0.7943 & 2.0249 & 0.031 & 0.0315 & 0.0009 & 0.001 & 0.0057 & 0.04 & 0.0192 & 0 & 0.039 \\
\hline 5 & $1400 \mathrm{~K}$ & 1000 & 0.7332 & 2.0306 & 0 & 0 & 0 & 0.0949 & 0.0526 & 0.0677 & 0 & 0 & 0.0062 \\
\hline 6 & $1600 \mathrm{~K}$ & 1000 & 0.5967 & 1.9687 & 0.0291 & 0.0094 & 0 & 0.1126 & 0.1108 & 0.0729 & 0.0231 & 0 & 0.0082 \\
\hline 7 & $1800 \mathrm{~K}$ & 1000 & 0.354 & 1.9078 & 0.032 & 0.0383 & 0.0354 & 0.2051 & 0.1507 & 0.1168 & 0.0181 & 0.0017 & 0.0047 \\
\hline 8 & $2000 \mathrm{~K}$ & 1000 & 0.2803 & 1.7854 & 0.044 & 0.0289 & 0.006 & 0.1978 & 0.2636 & 0.0993 & 0.0364 & 0 & 0.0023 \\
\hline 9 & $2200 \mathrm{~K}$ & 1000 & 0.2106 & 1.8528 & 0.0561 & 0.0728 & 0.0139 & 0.2126 & 0.2673 & 0.0373 & 0.0532 & 0.0001 & 0.0025 \\
\hline 10 & $2400 \mathrm{~K}$ & 1000 & 0.1323 & 1.6795 & 0.0538 & 0.0654 & 0.0041 & 0.2184 & 0.3331 & 0.0132 & 0.0018 & 0.0002 & 0.0018 \\
\hline 11 & $2600 \mathrm{~K}$ & 1000 & 0.1433 & 1.7387 & 0.0419 & 0.0588 & 0.0007 & 0.2083 & 0.2986 & 0.0093 & 0.0057 & 0.0006 & 0.0016 \\
\hline 12 & $2800 \mathrm{~K}$ & 1000 & 0.1062 & 1.6925 & 0.0219 & 0.0706 & 0.0014 & 0.1996 & 0.3136 & 0.0158 & 0.0008 & 0.0002 & 0.0013 \\
\hline 13 & $3000 \mathrm{~K}$ & 1000 & 0.0898 & 1.7053 & 0.0302 & 0.0864 & 0.0057 & 0.1466 & 0.1751 & 0.011 & 0.0045 & 0 & 0.0019 \\
\hline 14 & & & 0.4798 & 1.8598 & 0.0259 & 0.033 & 0.0049 & 0.1153 & 0.1408 & 0.0482 & 0.0116 & 0.0002 & 0.0209 \\
\hline
\end{tabular}




\begin{tabular}{l} 
Table S9. DeepMD simulation results for Pd nanocatalyst starting with 32 N-oxidoaniline and $64 \mathrm{H}_{2}$ molecules in a periodic cube with a side length of $3 \mathrm{~nm}$ with a 10 \\
ps time scale. \\
\hline
\end{tabular}




\begin{tabular}{|c|c|c|c|c|c|c|c|c|c|c|}
\hline & tmpr & ntraj & $\mathrm{H} 2$ & $\mathrm{O} 2$ & $\mathrm{H}$ & $\mathrm{HO}$ & $\mathrm{H} 2 \mathrm{O}$ & N4 & O6 & $\mathrm{O}$ \\
\hline 0 & $400 \mathrm{~K}$ & 1000 & 0.9844 & 0.1004 & 0.0312 & 0 & 0 & 0 & 0.0014 & 0.6904 \\
\hline 1 & $600 \mathrm{~K}$ & 1000 & 0.9844 & 0.0907 & 0.0312 & 0 & 0 & 0 & 0.0025 & 0.7179 \\
\hline 2 & $800 \mathrm{~K}$ & 1000 & 0.9844 & 0.1064 & 0.0312 & 0 & 0 & 0 & 0.0022 & 0.6917 \\
\hline 3 & $1000 \mathrm{~K}$ & 1000 & 0.9844 & 0.0689 & 0.0197 & 0.0111 & 0 & 0 & 0.0008 & 0.793 \\
\hline 4 & $1200 \mathrm{~K}$ & 1000 & 0.9844 & 0.0606 & 0.0122 & 0.0189 & 0 & 0 & 0.0011 & 0.8116 \\
\hline 5 & $1400 \mathrm{~K}$ & 1000 & 0.9844 & 0.0565 & 0.0287 & 0.0026 & 0 & 0 & 0.0011 & 0.8401 \\
\hline 6 & $1600 \mathrm{~K}$ & 1000 & 0.9844 & 0.0799 & 0.021 & 0.0102 & 0 & 0 & 0.001 & 0.7837 \\
\hline 7 & $1800 \mathrm{~K}$ & 1000 & 0.9844 & 0.0622 & 0.0301 & 0.0011 & 0 & 0 & 0.0011 & 0.8353 \\
\hline 8 & $2000 \mathrm{~K}$ & 1000 & 0.9899 & 0.0431 & 0.0065 & 0.012 & 0.0008 & 0 & 0.0005 & 0.868 \\
\hline 9 & $2200 \mathrm{~K}$ & 1000 & 0.9608 & 0.0489 & 0.0115 & 0.0348 & 0.0136 & 0 & 0.0004 & 0.8157 \\
\hline 10 & $2400 \mathrm{~K}$ & 1000 & 0.9294 & 0.0604 & 0.0354 & 0.0917 & 0.0068 & 0 & 0.0002 & 0.7561 \\
\hline 11 & $2600 \mathrm{~K}$ & 1000 & 0.9206 & 0.0464 & 0.0293 & 0.1176 & 0.0046 & 0 & 0.0003 & 0.7506 \\
\hline 12 & $2800 \mathrm{~K}$ & 1000 & 0.8598 & 0.0305 & 0.0686 & 0.1096 & 0.0504 & 0 & 0.0001 & 0.7529 \\
\hline 13 & $3000 \mathrm{~K}$ & 1000 & 0.8807 & 0.0382 & 0.0849 & 0.1085 & 0.0223 & 0 & 0.0001 & 0.7756 \\
\hline 14 & $3200 \mathrm{~K}$ & 1000 & 0.8604 & 0.0529 & 0.0841 & 0.1408 & 0.0254 & 0 & 0.0003 & 0.7058 \\
\hline 15 & $3400 \mathrm{~K}$ & 1000 & 0.8277 & 0.0486 & 0.1177 & 0.1471 & 0.0376 & 0 & 0.0001 & 0.694 \\
\hline 16 & $3600 \mathrm{~K}$ & 1000 & 0.7773 & 0.0335 & 0.1299 & 0.2115 & 0.0507 & 0 & 0.0002 & 0.648 \\
\hline 17 & $3800 \mathrm{~K}$ & 1000 & 0.7006 & 0.0353 & 0.1918 & 0.2638 & 0.0678 & 0 & 0.0003 & 0.569 \\
\hline 18 & $4000 \mathrm{~K}$ & 1000 & 0.6507 & 0.034 & 0.2595 & 0.2728 & 0.0797 & 0 & 0.0003 & 0.5575 \\
\hline 19 & & & 0.907 & 0.0578 & 0.0644 & 0.0818 & 0.0189 & 0 & 0.0007 & 0.7398 \\
\hline
\end{tabular}

